\title{
A NOVEL FPGA IMPLEMENTATION OF HIERARCHICAL TEMPORAL MEMORY SPATIAL POOLER
}

by

Paul Jeffrey Mitchell

\author{
A thesis \\ submitted in partial fulfillment \\ of the requirements for the degree of \\ Master of Science in Electrical \& Computer Engineering \\ Boise State University
}

December 2018 
(C) 2018

Paul Jeffrey Mitchell ALL RIGHTS RESERVED 


\title{
DEFENSE COMMITTEE AND FINAL READING APPROVALS
}

\author{
of the thesis submitted by
}

Paul Jeffrey Mitchell

Thesis Title: A Novel FPGA Implementation of Hierarchical Temporal Memory Spatial Pooler

Date of Final Oral Examination: $\quad 30$ October 2018

The following individuals read and discussed the thesis submitted by student Paul Jeffrey Mitchell, and they evaluated the student's presentation and response to questions during the final oral examination. They found that the student passed the final oral examination.

Nader Rafla, Ph.D. Chair, Supervisory Committee

Jennifer A. Smith, Ph.D. Member, Supervisory Committee

Hao Chen, Ph.D. Member, Supervisory Committee

The final reading approval of the thesis was granted by Nader Rafla, Ph.D., Chair of the Supervisory Committee. The thesis was approved by the Graduate College. 


\section{DEDICATION}

I dedicate this work to my two beautiful girls, Wendy and Lilly, and to my angel

wife April, for giving up those precious nights and weekends with "Daddy." Your sacrifices and support mean the world to me. 


\section{ACKNOWLEDGEMENTS}

I'd like to acknowledge Nader Rafla, PhD., Jennifer Smith PhD., and Hao Chen $\mathrm{PhD}$., for their support and flexibility while serving on my committee. Also, I would like to recognize Keith Roskelly, Neil Panchal, and all of the other members of the teams I've worked with at Apple and Marvell while working on this thesis. My managers, as well as

my teammates, have shown an overwhelming level of support, and have all sacrificed to some extent to allow me to pursue this work. 


\begin{abstract}
There is currently a strong focus across the technological landscape to create machines capable of performing complex, objective based tasks in a manner similar to, or superior to a human. Many of the methods being explored in the machine intelligence space require large sets of labeled data to first train, and then classify inputs. Hierarchical Temporal Memory (HTM) is a biologically inspired machine intelligence framework which aims to classify and interpret streaming unlabeled data, without supervision, and be able to detect anomalies in such data.

In software HTM models, increasing the number of "columns" or processing elements to the levels required to make meaningful predictions in complex data can be prohibitive to analyzing in real time. There exists a need to improve the throughput of such systems. HTMs require large amounts of data available to be accessed randomly, and then processed independently. FPGAs provide a reconfigurable, and easily scalable platform ideal for these types of operations. One of the two main components of the HTM architecture is the "spatial pooler". This thesis explores a novel hardware implementation of an HTM spatial pooler, with a "boosting" algorithm to increase homeostasis, and a novel classification algorithm to interpret input data in real time. This implementation shows a significant speedup in data processing, and provides a framework to scale the implementation based on the available hardware resources of the FPGA.
\end{abstract}




\section{TABLE OF CONTENTS}

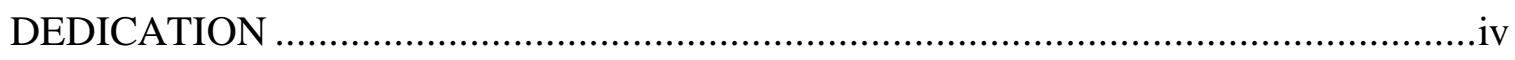

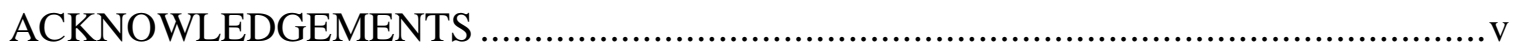

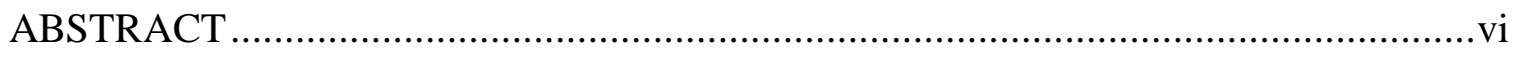

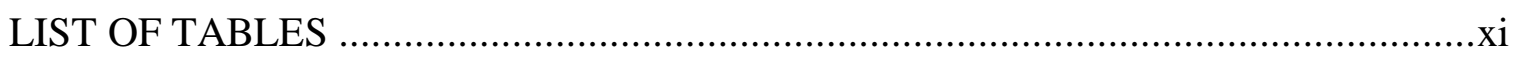

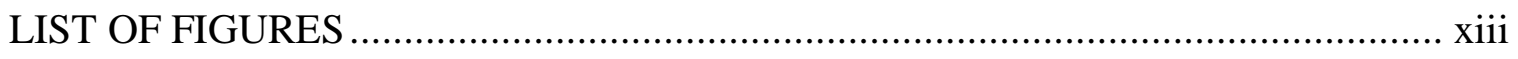

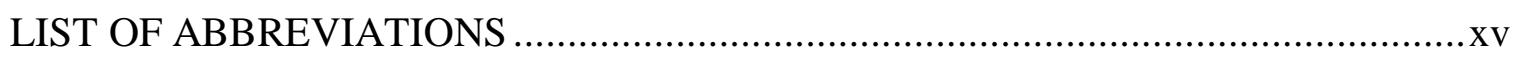

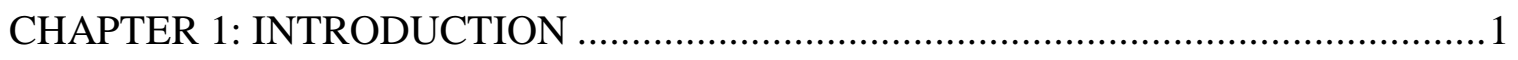

1.1 Motivation: The Demand for Smarter Machines ....................................1

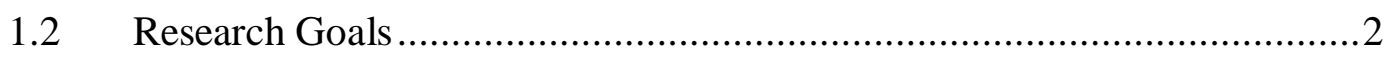

$1.3 \quad$ Evaluation Methodology ...................................................................

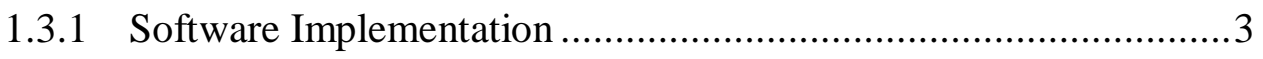

1.3.2 Verilog and Simulation ........................................................

1.3.3 FPGA Implementation ..........................................................

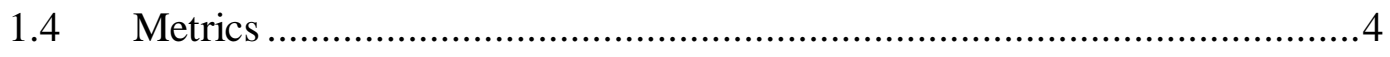

1.4.1 HTM Spatial Pooler ..............................................................

1.4.2 Classification Algorithm ……………………….....................

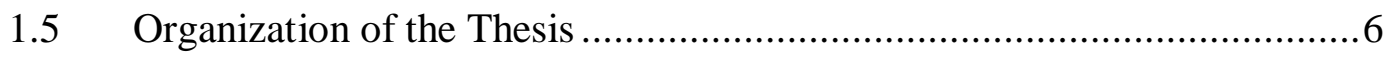

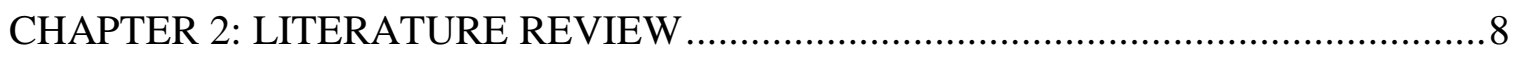

2.1 Hierarchical Temporal Memory ........................................................ 


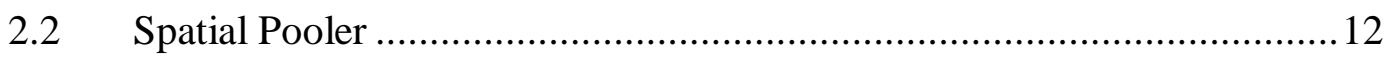

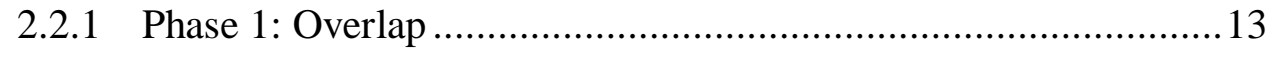

2.2.2 Phase 2: Winner Selection..........................................................13

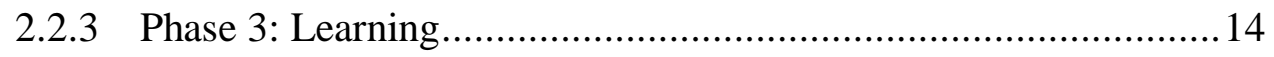

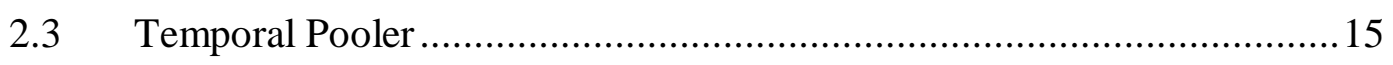

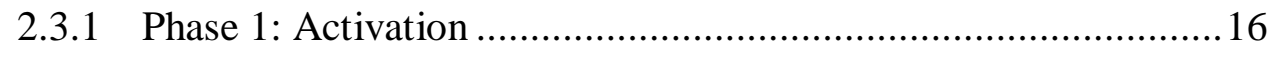

2.3.2 Phase 2: Overlap / Prediction .................................................... 17

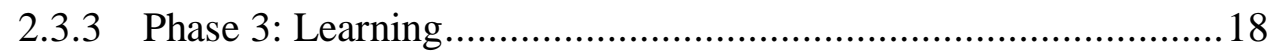

2.4 Classification of Sparsely Distributed Representations.............................19

2.4.1 Support Vector Machines ……………………….....................20

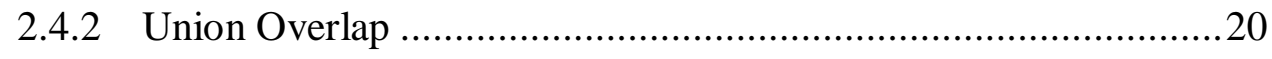

$2.5 \quad$ HTM Spatial Pooler Hardware Implementations ....................................22

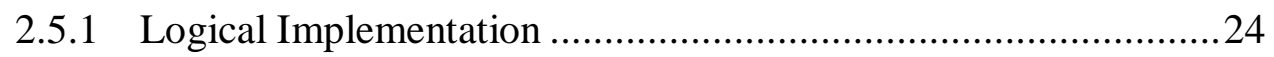

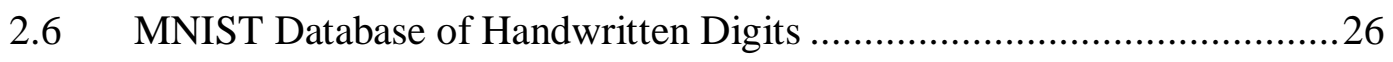

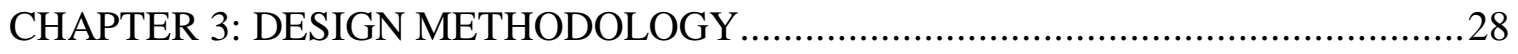

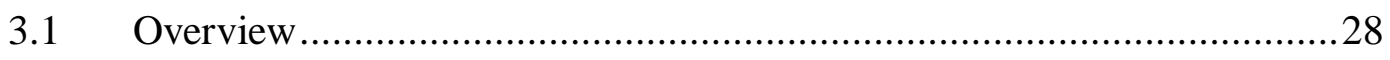

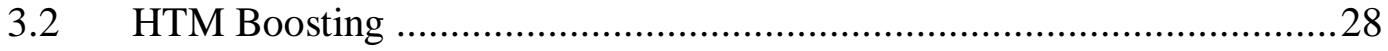

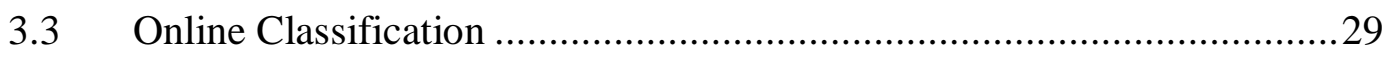

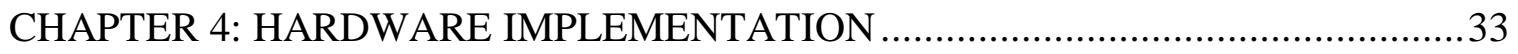

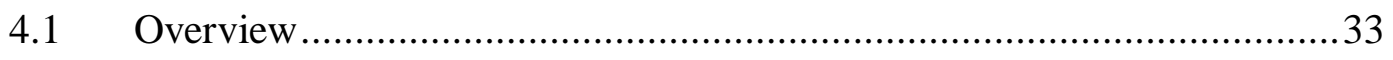

4.2 Primary Finite State Machine …………………....................................34

4.3 Linear Feedback Shift Register ...........................................................36

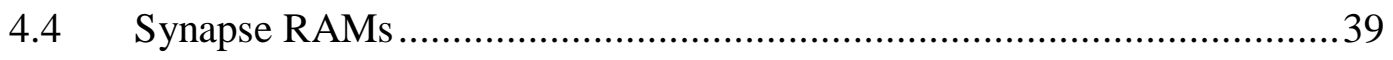


4.5 Winner Selection State Machine ..................................................40

4.6 Classification Logic ............................................................... 44

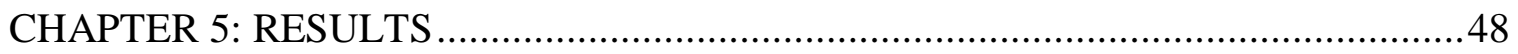

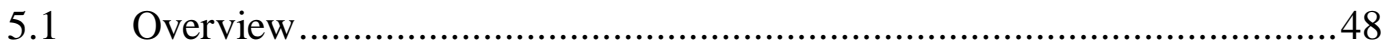

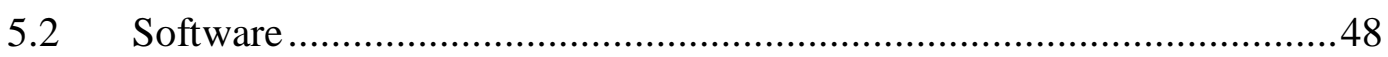

5.2.1 Number of Columns .........................................................50

5.2.2 Synapses per Column ......................................................51

5.2.3 Permanence Threshold ...........................................................51

5.2.4 Inhibition Radius .......................................................52

5.2.5 Variable Minimum Overlap ............................................53

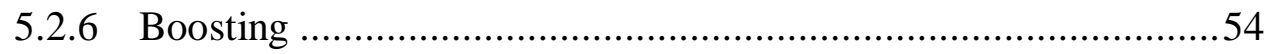

5.2.7 Minimum Overlap.............................................................55

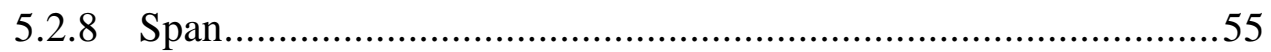

5.2.9 Final Parameters for Hardware Configuration .........................56

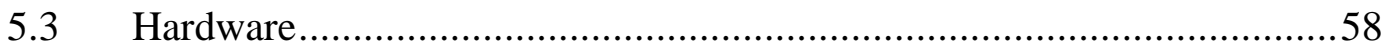

5.3.1 Classification Methods Results ............................................59

5.3.2 Hardware Parameter Sweep ..................................................59

5.3.3 FPGA Resources ............................................................6 61

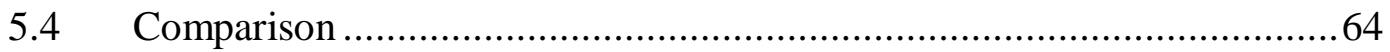

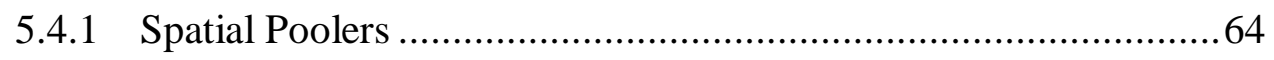

5.4.2 Classifiers ....................................................................6 65

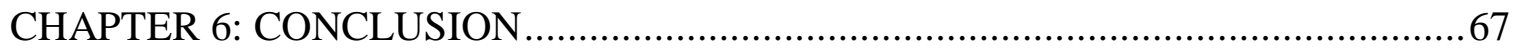

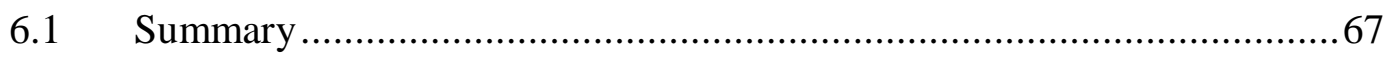




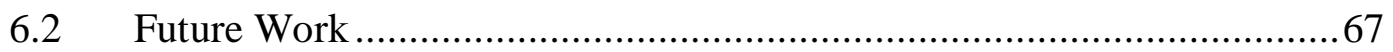

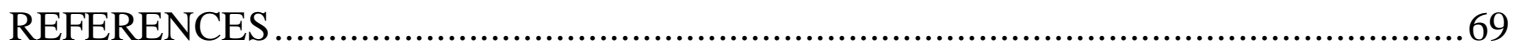

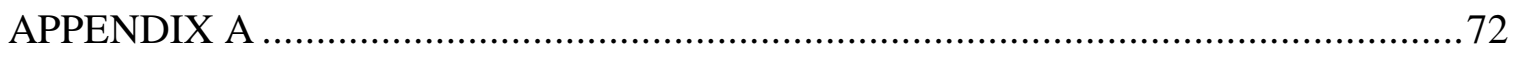

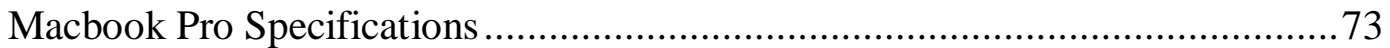

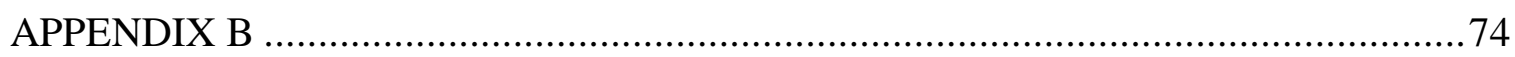

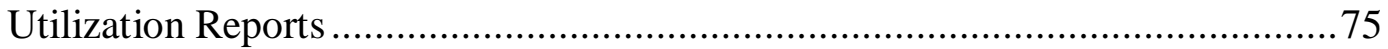




\section{LIST OF TABLES}

Table 1: $\quad$ Parameters used for Spatial Pooler (from Zyarah \& Dhireesha, 2015) .....23

Table 2. Classification registers initial values .............................................46

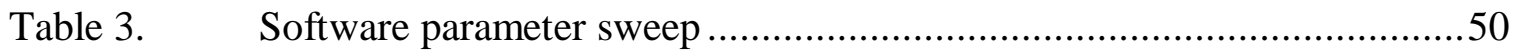

Table 4. Number of columns software sweep ...........................................51

Table 5. Synapses per column software sweep ..........................................51

Table 6. Permanence threshold software sweep ............................................52

Table $7 . \quad$ Inhibition radius software sweep................................................53

Table 8. Variable min overlap software sweep ..........................................54

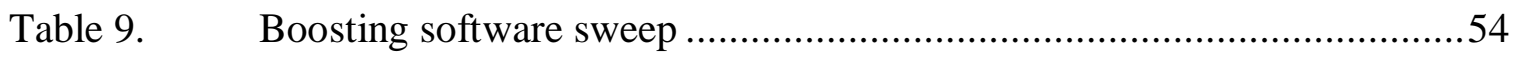

Table 10. $\quad$ Minimum overlap software sweep .............................................55

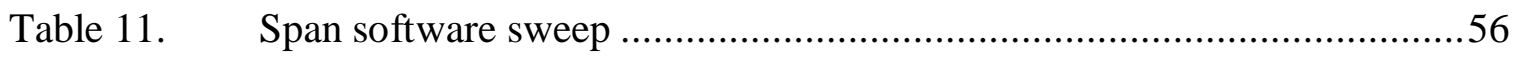

Table 12. Final software experiment parameters .........................................57

Table 13. Final software experiment results ...............................................57

Table 14. Scaled union overlap versus union overlap ....................................58

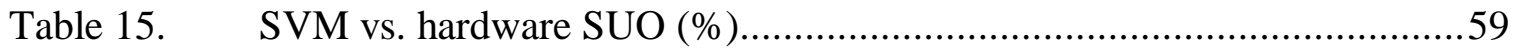

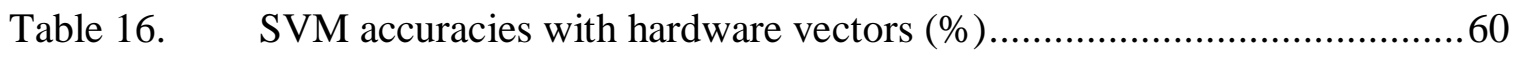

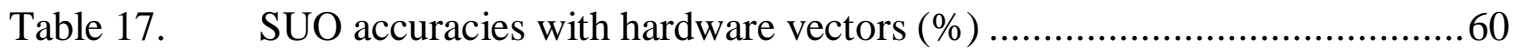

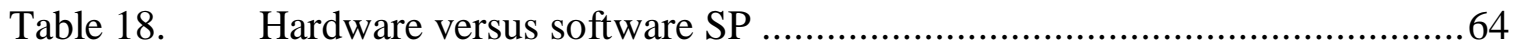

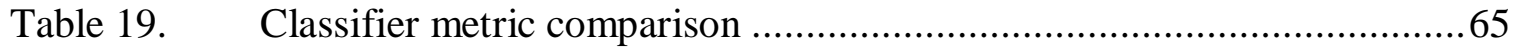


Table B.1 All functions enabled LUT utilization......................................... 75

Table B.2 All functions enabled RAM utilization ............................................. 75

Table B.3 All functions enabled DSP utilization .............................................. 76

Table B.4 Boosting disabled LUT utilization .................................................. 76

Table B.5 Boosting disabled RAM utilization ............................................... 77

Table B.6 Boosting disabled DSP utilization................................................... 77

Table B.7 Classification disabled LUT utilization .............................................. 77

Table B.8 Classification disabled RAM utilization............................................. 78

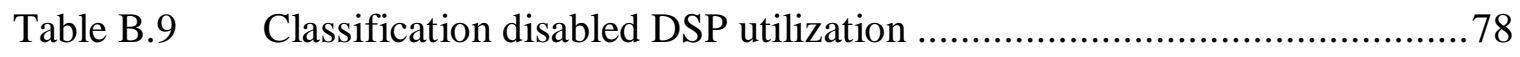




\section{LIST OF FIGURES}

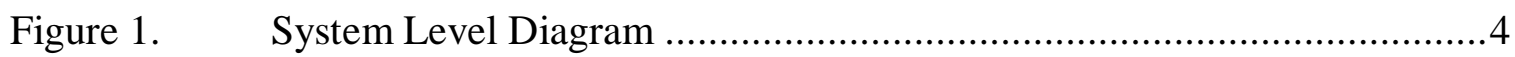

Figure 2. Levels of hierarchy in an HTM (from Numenta, 2011) .........................10

Figure 3. Multi-input HTM network (from Numenta, 2011) …..............................11

Figure 4. Topology of an HTM region (from Numenta, 2011) ..............................12

Figure 5. SP Phase 1 pseudo-code (from Numenta, 2011)......................................13

Figure 6. SP Phase 2 pseudo-code (from Numenta, 2011).....................................14

Figure 7. SP Phase 3 pseudo-code (from Numenta, 2011)....................................15

Figure 8. Temporal pooling (from Numenta, 2011)..........................................17

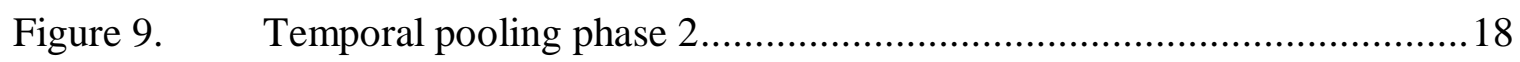

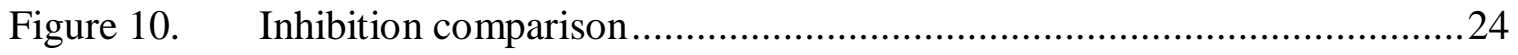

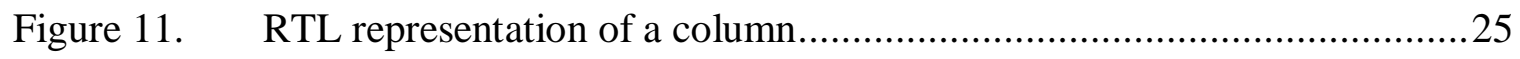

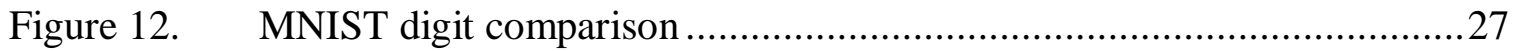

Figure 13. Scaled Union Overlap vs. regular union overlap ……………….............30

Figure 14. Scaled Union Overlap pseudo-code ……….............................................

Figure 15. Modified scaled union overlap pseudo-code ………................................32

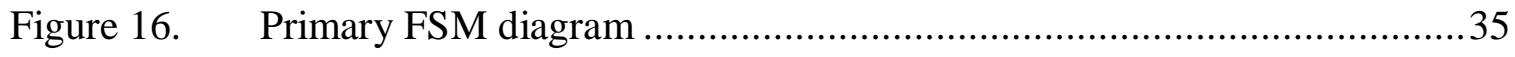

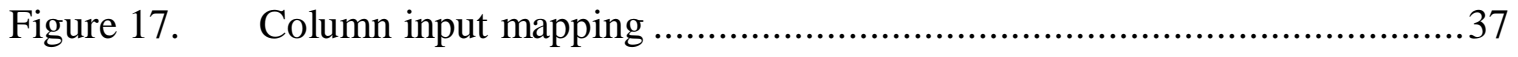

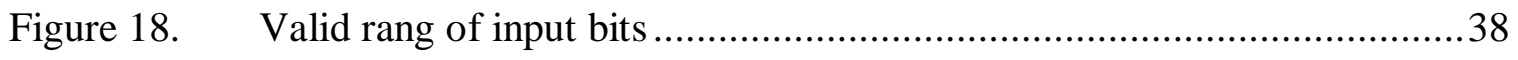

Figure 19. Linear feedback shift register diagram ..................................................38 


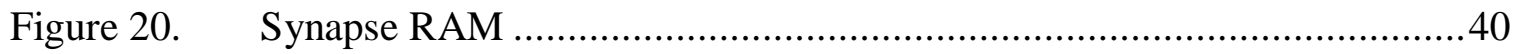

Figure 21. Winner selection SM diagram...................................................... 41

Figure 22. Duty cycle computation ............................................................. 44

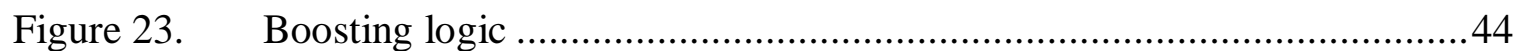

Figure 24. Union vector SRAM ............................................................. 47

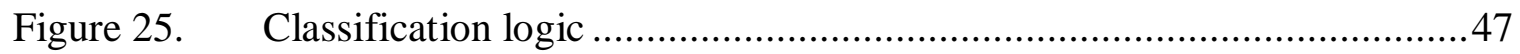

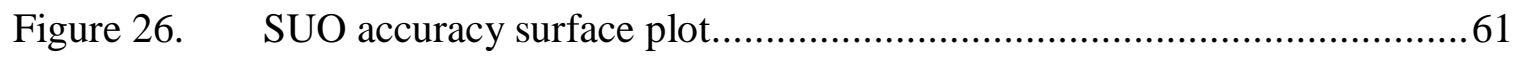

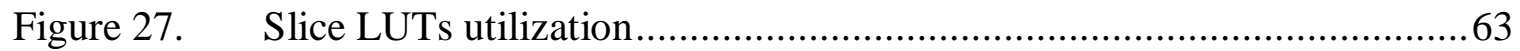




\section{LIST OF ABBREVIATIONS}

HTM

SDR

ML

AI

FPGA

ASIC

SVM

UO

SUO

HDL

CPU

GPU

LUT

DSP

RAM

SRAM

SP

TP

LFSR

MNIST
Hierarchical Temporal Memory

Sparsely Distributed Representation

Machine Learning

Artificial Intelligence

Field Programmable Gate Array

Application Specific Integrated Circuit

Support Vector Machine

Union Overlap

Scaled Union Overlap

Hardware Description Language

Central Processing Unit

Graphical Processing Unit

Look-Up Table

Digital Signal Processor/Processing

Random Access Memory

Static Random Access Memory

Spatial Pooler

Temporal Pooler

Linear Feedback Shift Register

Modified National Institute of Standards and Technology 
Register Transfer Level 


\section{CHAPTER 1: INTRODUCTION}

\subsection{Motivation: The Demand for Smarter Machines}

The field of artificial intelligence has been explored since the mid 1950's, but has made a recent resurgence into mainstream society [1]. Technology companies have begun marketing their products based on their use of hardware designed specifically to perform Artificial Intelligence (AI) tasks. Throughout 2016 and 2017, the technology company NVIDIA has pivoted their primary focus from video gaming hardware, to hardware accelerated neural networks. Apple has led the smartphone industry by incorporating dedicated neural network hardware directly on their SoCs in their 2017 flagship products. Qualcomm has followed suit and included similar hardware in some of their top-of-theline SoCs. Even enterprise level datacenters are moving to using ASICs and reconfigurable hardware to implement machine learning algorithms for the performance, cost, and environmental benefits [2].

There is a broad range of applications for which machine learning provides both increased performance and reduced energy consumption. It's estimated that electricity accounts for about $15 \%$ of a datacenter's operational cost [3]. Consequently, measurable decreases in computational energy consumption can have a significant impact on the bottom line for a company, which processes large quantities of data. Machine learning algorithms are general learning algorithms which can be applied to datasets with vastly differing domains. So, one algorithm, if implemented in hardware, could be used in a wide range of applications. As more and more computationally intensive tasks move to 
using machine learning, the case becomes stronger to utilize specialized hardware for this purpose.

Many machine learning, more specifically deep learning, algorithms require a large number of simple operations on large sets of matrices during training. Consequently, GPUs are a popular choice as an alternative to commercially available CPUs for performing these computations. For instance, the popular machine learning platform TensorFlow from Google utilizes GPUs for training many of its machine learning models [4]. Whereas GPUs do provide several benefits over CPUs [5], like performance per core, and performance per Watt, when it comes to performing only one specific type of algorithm, an FPGA or ASIC in many cases will provide even greater performance, while using less power. [6, 7]

\subsection{Research Goals}

This thesis will describe and evaluate the effectiveness of a novel implementation

of the Hierarchical Temporal Memory (HTM) spatial pooler model [8] on reconfigurable hardware. The novel elements include:

1. Pipelining between the "Phase 1" and "Phase 2" portions of the algorithm 2. FPGA implementation of the HTM "boosting" algorithm, and

3. Development and implementation of a new online classification algorithm called "Scaled Union Overlap"

To achieve this goal, the HTM algorithm was first built in software and parameterized, and then translated to synthesizable Verilog, a common hardware description language [9]. This model was then simulated and verified before implementing it on a Xilinx 7-series Zynq FPGA development board [10]. Finally, the 
MNIST dataset [11] was used to verify the functionality of the hardware and its ability to implement the HTM algorithm as well as the classifier's accuracy relative to conventional software means.

\subsection{Evaluation Methodology}

This section describes the strategy which was used to evaluate the performance of the novel components of this work. In addition to the steps taken, the software and HDL tools are presented.

\subsubsection{Software Implementation}

To establish the effectiveness of the HTM algorithm to be implemented on the FPGA, it was built, verified, and validated in software using Python for its ease of development, object-oriented nature, and speed. On the other hand, the new classification algorithm was implemented using a Perl script. A support vector machine library called LIBSVM [12] was used to validate the effectiveness of the spatial pooler's ability to extract meaningful features, and to benchmark the new classification algorithm.

\subsubsection{Verilog and Simulation}

Verilog HDL will be used to create a synthesizeable version of the spatial pooler and classification algorithm. The Xilinx IDE Vivado will serve as the synthesis / PNR tool. Simulations were run with the open source software Icarus [13] for its cross platform support, and waveforms observed with GTKWave [14]. Simulations were only used to verify the HDL implementation, and were not used to provide any metrics for evaluation of the effectiveness of the algorithms. 


\subsubsection{FPGA Implementation}

The HTM spatial pooler RTL was constructed as an AXI peripheral, and controlled by the Zynq processing system. Training data was provided via an SD card, and status communicated via UART to a terminal on an external machine. The output of the system was written to an SD card, and transferred to a Macbook Pro for analysis (see Appendix A for specifications). Figure 1 illustrates the connectivity between the HTM core and the external system.

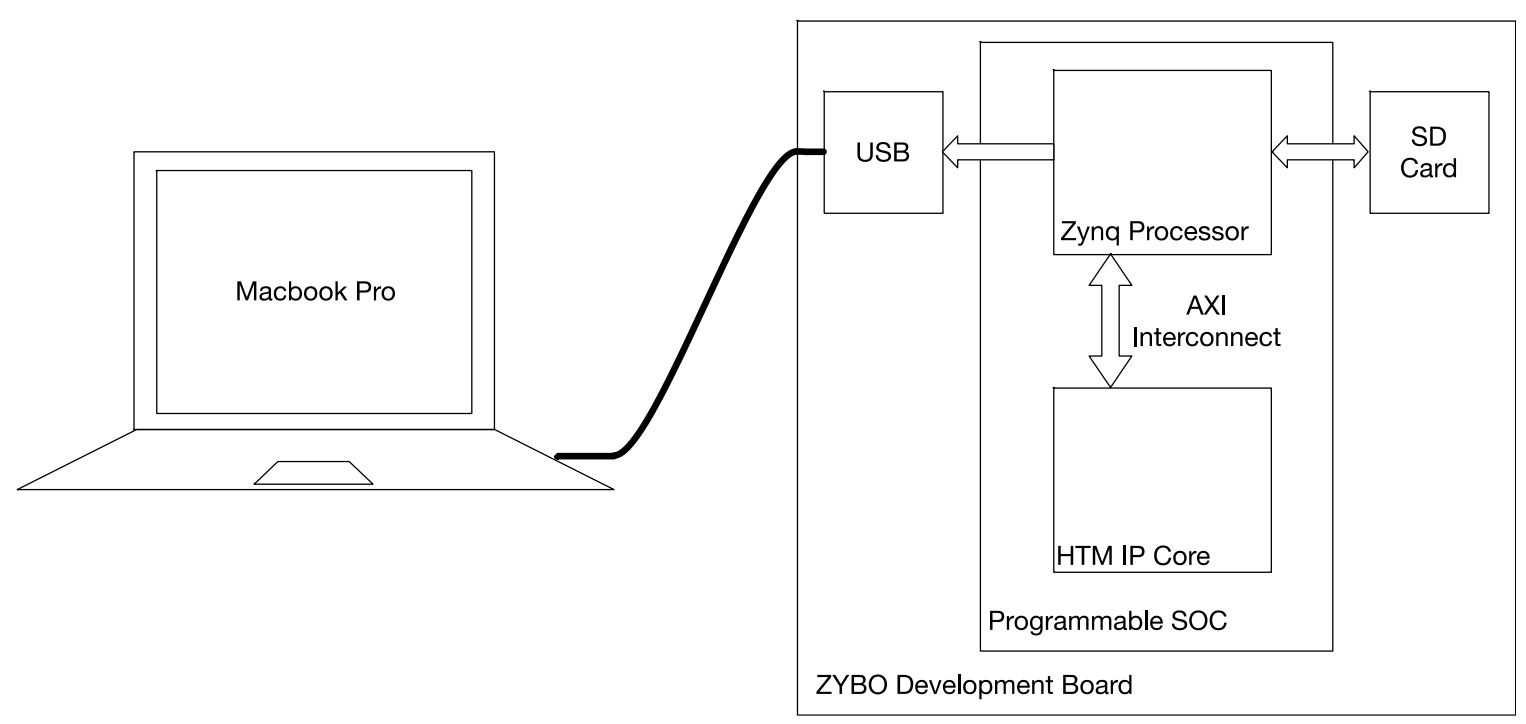

Figure 1. System Level Diagram

\subsection{Metrics}

\subsubsection{HTM Spatial Pooler}

The spatial pooler was evaluated based on four primary metrics: speed, energy consumption, resources, and feature extraction. Speed is measured by the number of training samples the spatial pooler will be able to process each second, and is denoted by $f\left(s_{t}, t\right)$ as shown in equation 1.

$$
f\left(s_{t}, t\right)=\frac{\text { number of samples }}{\text { training time }}=\frac{s_{t}}{t}
$$


The speeds for the software and hardware implementations are calculated using equation 1 , and compared.

Energy consumption is measured in Joules per sample, and is calculated based on the total training time, number of training samples, and average power.

$$
E\left(P_{\text {ave }}, t, s_{t}\right)=\frac{\text { average power } \times \text { training time }}{\text { number of samples }}=\frac{P_{\text {ave }} t}{s_{t}}
$$

The average power used for the software simulation is found by taking the average power consumed while simulating the HTM on the Macbook, and subtracting the average power measured while the system is idle. For comparison, the average power for the FPGA based solution is measured directly. In both cases, the same power measurement device is used, which has a claimed typical error rate of $0.5 \%$ [15].

The resources metric only applies to the hardware implementation, and is a measure of the physical resources used by the FPGA implementation. These resources consist of: (a) number of lookup tables (LUTs), (b) number of slice registers (slices), (c) number of DSP slices, and (d) number of block RAMs used. The feature extraction metric is an indication of the ability of the system to encode the features of a dataset in a meaningful way. It is represented as a percentage of correctly classified samples, and is used to compare both the hardware and software implementations.

\subsubsection{Classification Algorithm}

The hardware classification algorithm is compared to the software classification with speed, energy consumption, and classification accuracy metrics. The speed and classification metrics are calculated similarly to that of the spatial pooler, except the 
difference in time and average power between experiments with both classification enabled and classification disabled is used.

$$
f_{c}\left(t, t_{c}, s\right)=\frac{\text { number of samples }}{\text { time } / \text { w classifying - time } / \text { wo classifying }}=\frac{s}{t_{c}-t}
$$

The value of $f_{c}$ represents the frequency in samples per second that the classifier can operate. If $P_{a v e, c}$ represents the average power consumed during classification, then the energy consumed classifying each sample can be written as:

$$
E_{c}\left(P_{a v e, c}, P_{a v e}, t_{c}, t, s\right)=\frac{P_{a v e, c} t_{c}-P_{a v e} t}{s}
$$

The final metric to compare classification algorithms is the classification accuracy. For a given set of vectors, each of which belong to one of a discreet set of classes, the classifier is measured by its ability to correctly identify to which class each vector belongs. The percentage of correctly identified vectors will indicate the effectiveness of the classification algorithm.

$$
A=100 \times \frac{\text { correctly identified vectors }}{\text { total vectors classified }}=100 \times \frac{c}{s_{c}}
$$

\subsection{Organization of the Thesis}

The rest of the thesis is organized as follows

- Chapter 2 discusses the related literature in the fields related to Hierarchical Temporal Memory, Spatial Pooler, and Temporal Pooler.

- Chapter 3 details the design methodology for the Hierarchical Temporal Memory Spatial Pooler.

- Chapter 4 covers the hardware implementation of the HTM on the selected FPGA platform. 
- Chapter 5 discusses the software and hardware results obtained along with the experiments, verification, and tests performed.

- Chapter 6 concludes the thesis and provides suggestions for future work. 


\section{CHAPTER 2: LITERATURE REVIEW}

\subsection{Hierarchical Temporal Memory}

Hierarchical Temporal Memory, or HTM, is a biologically inspired theory of intelligence maintained by the privately funded company Numenta. It is based on the cortical learning algorithm, or CLA, described by John Hawkins and Sandra Blakeslee in their book "On Intelligence" [16]. The basic algorithm involved in HTM falls within the deep learning family of machine learning. The goal of HTM is to model the operation of the mammalian neocortex, and in time to help researchers understand the nature of human intelligence through building machines capable of replicating it. The HTM spatial pooling algorithm was created by Numenta, and is described by Hawkins and Subutai in their whitepaper titled "Hierarchical Temporal Memory including HTM Cortical Learning Algorithms" [8].

The fundamental unit of the HTM model is a cell. A cell is an abstraction of a physical cell in the brain, or a neuron. HTM cells have many similarities with biological neurons, such as; they have feed forward, as well as lateral I/O connections, each is either "active" or "inactive" at a given point in time, the strength of the I/O connections is updated based on both current inputs, as well as previous inputs, and both exhibit a form of local inhibition to nearby cells. In the interest of simplifying the model, some characteristics of neurons are not present in HTM cells. For instance, HTM cells will typically only be connected to tens, or hundreds of other cells, whereas real neurons may have tens of thousands of connections each. 
Cells are arranged into groups called "columns". The name columns comes from the physical orientation most neurons have in the neocortex of mammalian brains. Columns are an abstraction for a group of cells, and are the fundamental unit of the HTM spatial pooler. Each cell in a particular column shares its feed forward input with that of the other cells in the same column. A column can be considered active if the number of connected active inputs is greater than a particular threshold, and that number is greater than that of all other columns in its inhibition radius.

Groups of cells which share a common input space, and which can inhibit one another, make up a region. The use of an activation threshold as well as local inhibition of cells ensures that regions should always maintain a sparsely distributed representation (SDR) of the input. An SDR is a vector in which relatively few elements are non-zero. HTM SDRs maintain a sparsity of about $10 \%$.

Regions can be grouped into hierarchies in which the output of each region (its activation pattern) is made to either be one of the inputs to another region, or the primary output of the system. Having multiple levels allows for greater levels of abstraction of the input data relative to the lower regions. This hierarchical structure is what qualifies HTM as a deep learning algorithm. All regions which exist in the same vertical position in the system hierarchy are in the same "level." Figure 1 shows a four level hierarchy, similar to the human neocortex, with the lines indicating the flow of temporal information. Lines between levels represent the feed-forward and feed-backward flow of data between regions. Lines within levels are the distal connections between cells. 


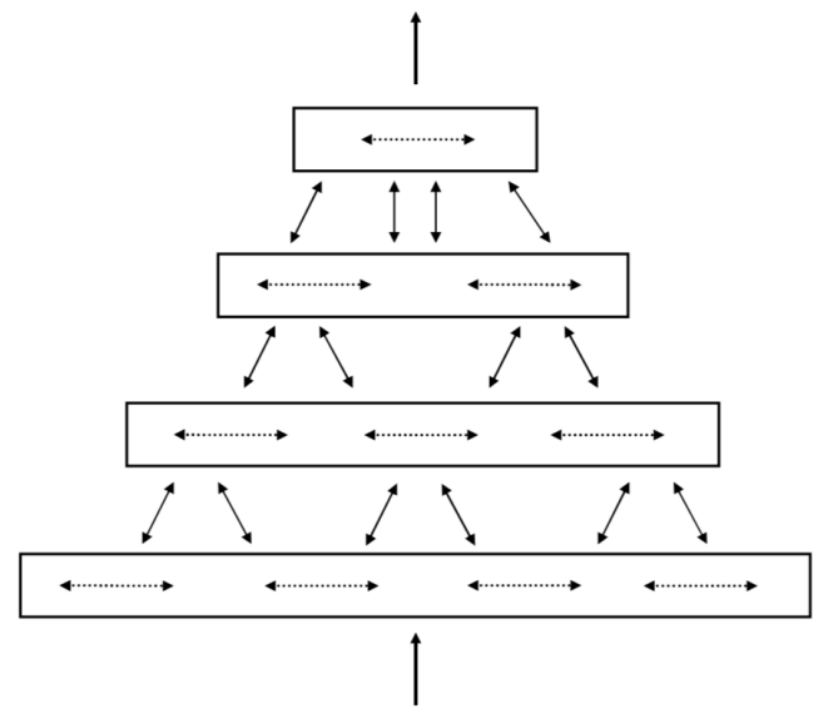

Figure 2. Levels of hierarchy in an HTM (from Numenta, 2011)

The system shown in figure 2 includes only one source, and only one output, in a pyramid structure. This type of structure where the regions decrease in size from input to output is a bottom-up network. The implementation described in this thesis will follow this topology. However, HTM isn't restricted to either a single input or a single output. Figure 3 shows a system which can take two streaming inputs, audio and video, extract features and patterns from each, and then classify and make predictions using both simultaneously. 


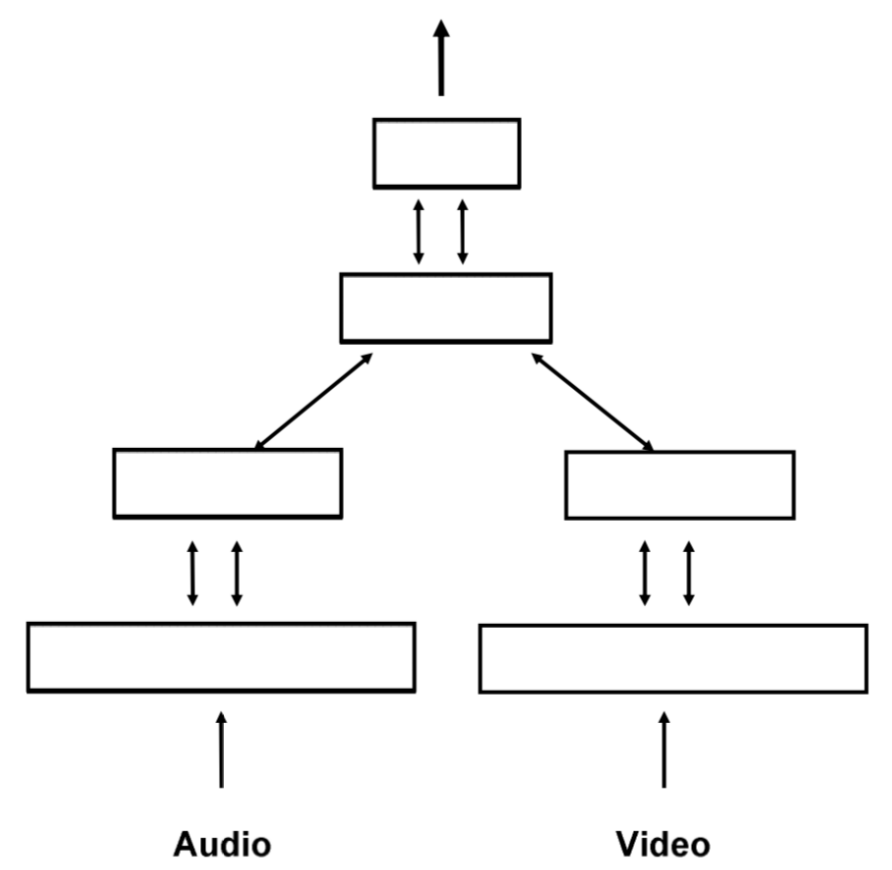

Figure 3. Multi-input HTM network (from Numenta, 2011)

Typically, HTM regions are two-dimensional arrays of columns. Using two dimensions of columns most closely resembles the structure seen in the neocortex, but the number of dimensions are not restricted to two. The implementation discussed in this work treats the columns as a one-dimensional array of columns. Each column consists of multiple cells. All cells within a column share the same feed-forward input, but each individual cell will have a large number of its own set of distal connections to other cells in the region. A column is considered active if at least one of its cells is active. The activation of columns indicates spatial information about the input, and the activation of the individual cells contains the temporal context.

Figure 4 shows an array of columns forming a small region, each with four cells. The activated cells are shown in dark blue. The number of unique temporal contexts $X$ able to be stored in a region for an activation pattern of columns can be found with equation 6. 


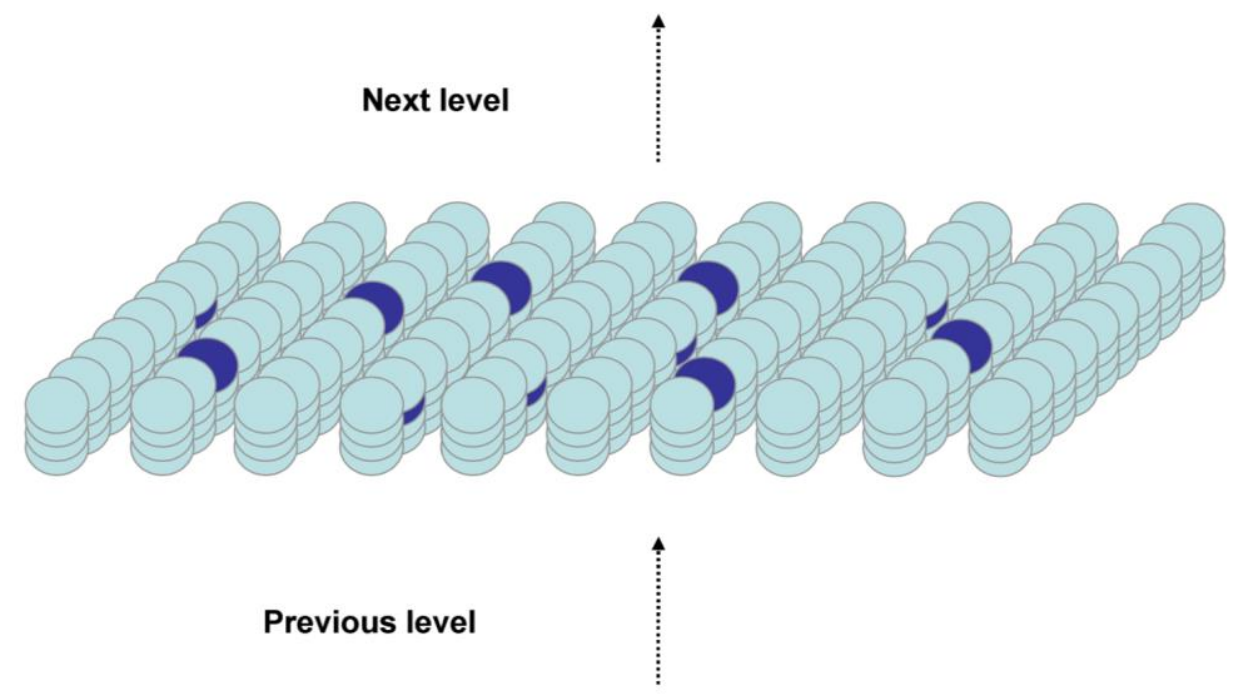

Figure 4. Topology of an HTM region (from Numenta, 2011)

$X=$ cells per column $n^{2}-2=c^{2}-2$

\subsection{Spatial Pooler}

The spatial pooler is responsible for determining which columns within a region will be active given the state of the input. Each column is connected to only a subset of the inputs to the region. This connection, also referred to as a synapse due to its biological counterpart, is represented with a scalar value called permanence. The permanence of a synapse is similar to the weights which are associated between connected processing elements in artificial neural networks. However, in the HTM model, synapses have only a binary weight, either 0 or 1 , based on the synapse's permanence being either above or below a certain threshold. Synapses below the threshold are labeled as "potential synapses", whereas those above the threshold are "functional synapses".

Initialization of the spatial pooler involves creating the initial feed forward synapses connecting each column to the input space for the region. Each column will have several synapses, each consisting of a pointer addressing the position within the 
input, as well as the initial permanence. The mean of the initial permanencies should be close to the connectivity threshold, giving roughly the same number of functional synapses as potential synapses.

\subsubsection{Phase 1: Overlap}

In the first phase of the spatial pooling algorithm, the activation, or overlap, of each column is computed by summing all synapses in which the synapse permanence is above the threshold, and the input bit is active. Optionally, the overlap may be scaled by a "boosting" factor. Figure 5 shows the pseudo-code for this phase.

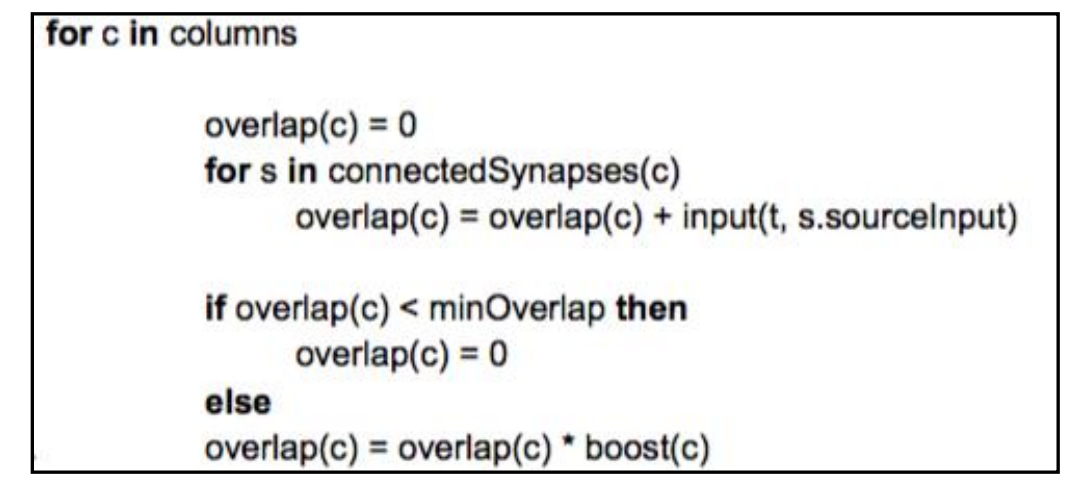

Figure 5. SP Phase 1 pseudo-code (from Numenta, 2011)

\subsubsection{Phase 2: Winner Selection}

Once all columns have the overlap computed, only those which have the highest local activation relative to the nearest neighboring columns are chosen as winners, and declared as active during that iteration. This resulting activation pattern is a sparsely distributed representation of the input. This is "Phase 2". The pseudo-code of phase 2 is depicted in Figure 6. 


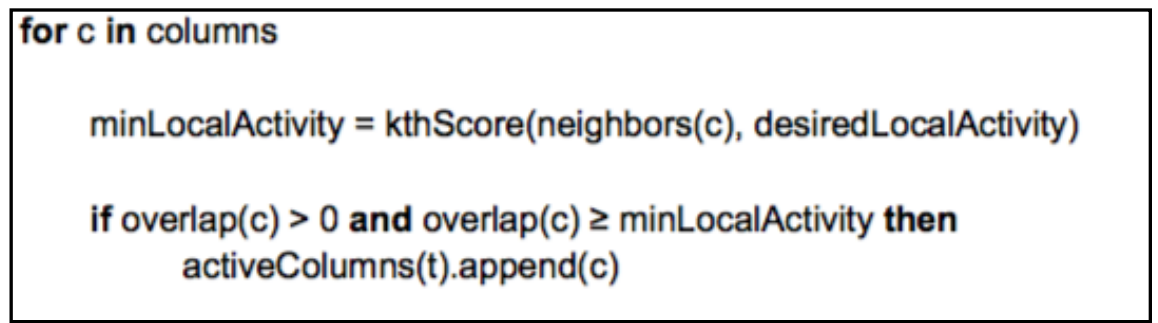

Figure 6. $\quad$ SP Phase 2 pseudo-code (from Numenta, 2011)

\subsubsection{Phase 3: Learning}

Once the winners are selected, the learning begins in "Phase 3". Learning occurs simply by adjusting the permanencies associated with each synapse of only the winning columns. For each synapse for every winning column, if the input bit was a 1 , the permanence is increased. If the input bit of the synapse was a 0 , the permanence is decreased. In this way, the correlation between the activation pattern and its corresponding input pattern is reinforced. The pseudo code of this phase is summarized in Figure 7. 


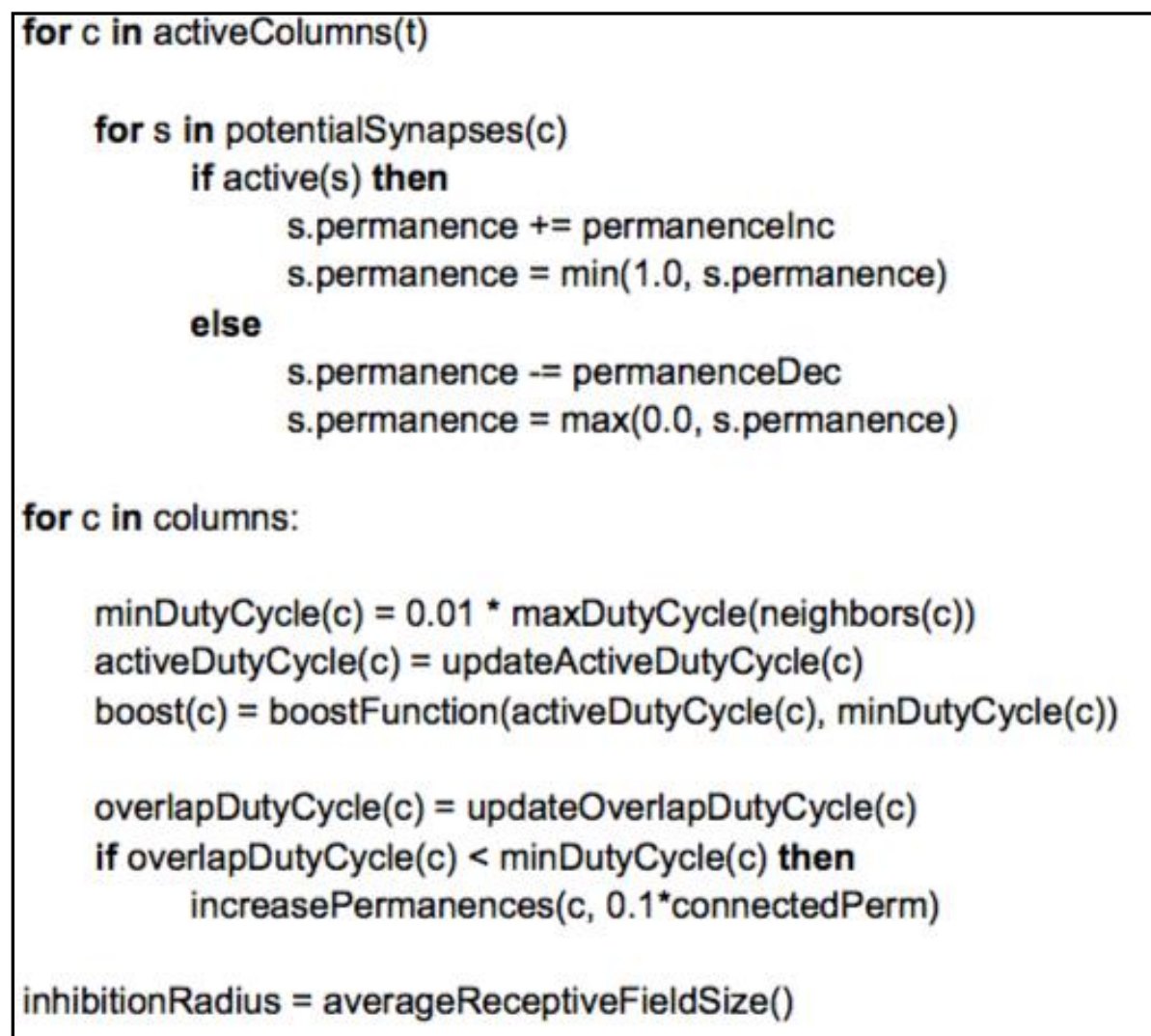

Figure 7. SP Phase 3 pseudo-code (from Numenta, 2011)

The first loop updates the permanence values for those columns which were

deemed as winners in Phase 2. The second loop updates the duty cycles for each column, and uses those duty cycles to compute the boost factors. HTM can be used either in an "online" or "offline" learning methodology by choosing whether or not Phase 3 occurs continuously, or is disabled after training is complete.

\subsection{Temporal Pooler}

The goal of temporal pooling is to identify and learn patterns in sequences of inputs. With the temporal pooler, it extends not only the ability of the HTM beyond just identifying features and classes of inputs, but also to predict future inputs from past patterns, based on the current temporal context of the current input. 
The temporal pooling algorithm begins after spatial pooling has completed, and is likewise separated into 3 phases. The mechanics of the temporal pooling algorithm operate similarly to the spatial pooling algorithm, with some additional complexities during the learning phase.

\subsubsection{Phase 1: Activation}

Temporal pooling begins where spatial pooling ends, with a set of active and inactive columns representing the current input. The temporal pooler will first determine which, if any, of the cells in the active columns are in a predictive state. The predictive state of the cells would have been set in phase 2 of the previous iteration of the temporal pooler. If no cells in an active column are in the predictive state, as is the case with all columns in the first iteration of the temporal pooler, then all of the cells in such a column are activated. When all of the cells in a column are activated, this represents an unexpected event, possibly a transition that has never been observed before by the HTM. When a large ratio of the active columns have all of their cells activated, an anomaly has occurred in the temporal sequence. In each column, a cell is selected as being the "learning cell" for phase 3 based on the activation of the previous time-step. The cell which contains the dendrite segment with the highest overlap with the previous activation is chosen to be the learning cell.

Figure 8 shows the state of an HTM with temporal pooling. The white cells are inactive, the dark grey cells represent a predictive state, and light grey indicates a cell is active for the current time-step. The columns in which all cells are light grey indicates a column was determined a winner by the spatial pooler, but no cells were in the predictive state prior to temporal pooling. 


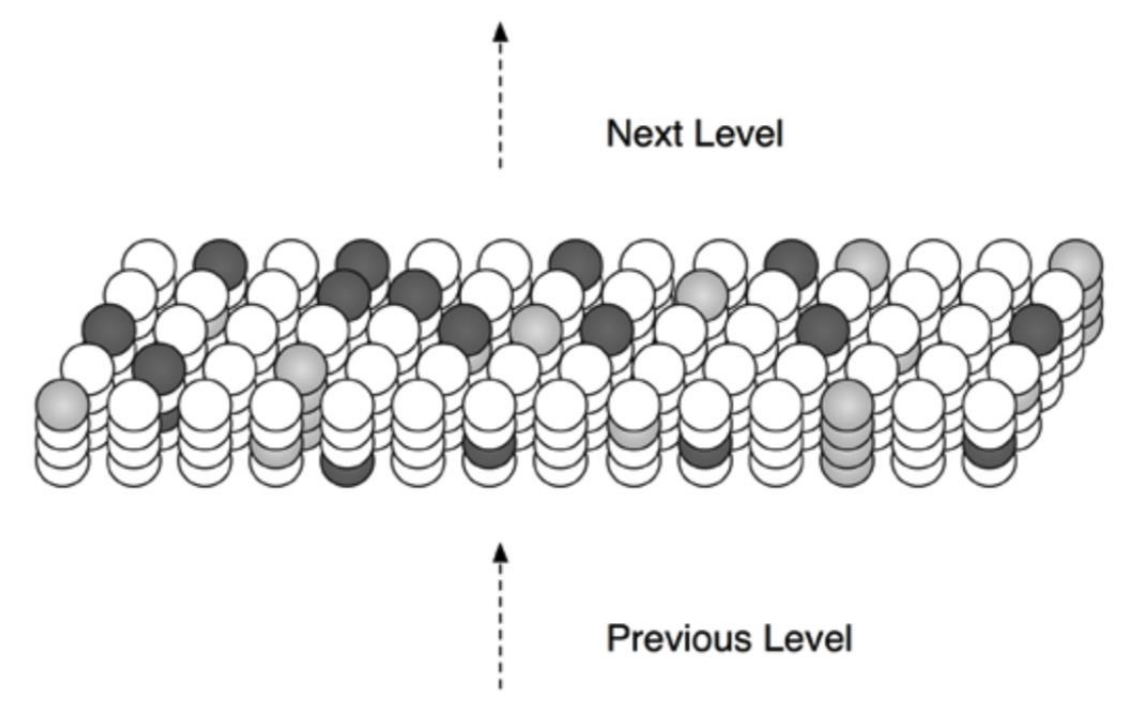

Figure 8. Temporal pooling (from Numenta, 2011)

\subsubsection{Phase 2: Overlap / Prediction}

Phase 2 of the temporal pooler is similar to the overlap phase of the spatial pooler.

Each cell has a number of its own synapses, but instead of being connected to the input space, these synapses are connected to other cells in the region. The synapses for a cell are grouped together into several "dendrite segments". The number of dendrite segments per cell is small in comparison to the number of synapses per dendrite segment. For each dendrite segment, the potential synapses are tested against a threshold. The synapses whose permanence is higher than the threshold are considered functional. The overlap of a dendrite segment is the sum of all of the functional synapses connected to cells in the active state. Here, the active state means a cell is active only due to feed forward input, and not simply in the predictive state. If the overlap of any of the dendrite segments for a cell is above a threshold, then that cell is put into the predictive state for the next time step.

Figure 9 illustrates the phase two prediction computation. In this example, the top cell in the center column is being tested to determine if it should be put into the predictive 
state. Each triangle represents a dendrite segment, and the lines from the dendrite segments to the other cells are functional synapses. A grey colored cell was deemed active during phase 1 of temporal pooling. The activation for dendrite segment A is two, and $\mathrm{D}$ is one. $\mathrm{B}$ and $\mathrm{C}$ are both zero. If the minimum activation for a dendrite segment were two or less, this cell would be placed in the predictive state for the next time-step.

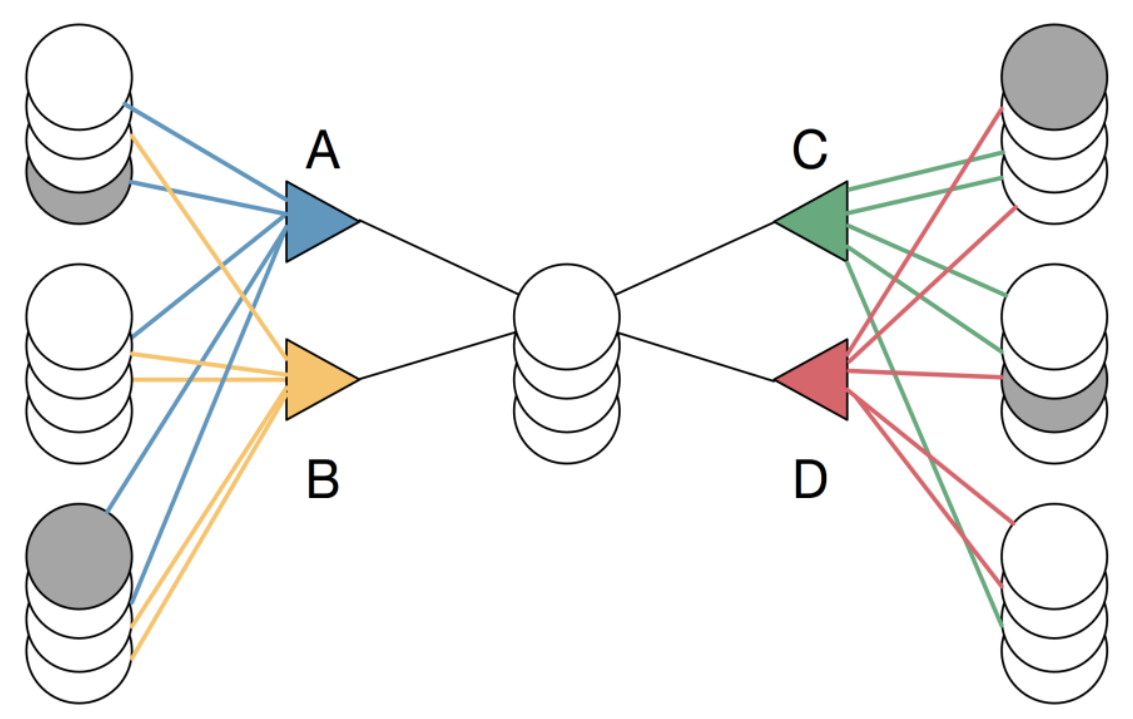

Figure 9. Temporal pooling phase 2

\subsubsection{Phase 3: Learning}

The learning phase of the temporal pooler iterates through every cell, and checks for two conditions:

1. If the cell was previously selected as the learning cell in its column, then each of the synapses on that learning cell are reinforced by increasing their permanence, but only if the synapse is connected to a cell which was activated in the previous time step.

2. If it was not a learning cell, and not currently in the predicted state, but in the previous time-step (predicted to be activated), then decrease the permanence of its distal connections. 
Case 1 reinforces the correlation between the previous state and the activation of the updated cells. The result being that the next time the activation pattern from the previous time step is seen, the current activation pattern will be predicted. Case 2 looks for instances where the previous iteration of the temporal pooler incorrectly predicted that a cell would become active, but it did not. Decreasing the permanencies for these cells decreases the likelihood of another false positive.

\subsection{Classification of Sparsely Distributed Representations}

A sparsely distributed representation is an encoding of data using a vector in which a small minority of its elements are non-zero. When the dimensionality of the vector is sufficiently large, there can be a relatively large number of possible encodings with only a small percentage of non-zero elements. For instance, a bit string of length 512 can represent more than 287 billion possible values when only 5 , or less than $1 \%$, of its bits are 1. The sparsity of an SDR is computed using the following equation.

$$
\text { Sparsity }=\frac{w}{n}
$$

where $\mathrm{n}$ is the total number of elements, and $\mathrm{w}$ is the number of non-zero elements. The number of unique encodings possible with an SDR is given by equation 8:

$$
\text { Unique SDR encodings }=\frac{n !}{w !(n-w) !}
$$

The human neocortex operates on SDRs when considering each neuron to be either on or off depending on its action potential [17]. The output of the spatial pooler is a sparsely distributed representation of the input features, and can be considered to be a "hidden layer" like in other deep learning algorithms. A hidden layer being a layer whose output does not directly translate to the system output, but must be processed further in order to provide meaningful information. Hierarchical Temporal Memory is in fact 
hierarchical in nature, and is intended to be used in multiple layers. As the number of layers' increases, so does the level of abstraction the SDRs can represent. However, to determine the efficacy of a single layer of the spatial pooler, its output needs to be classified using some other type of classification algorithm.

\subsubsection{Support Vector Machines}

Support vector machines (SVMs) aim to classify n dimensional data points by separating the data points using $\mathrm{n}$ dimensional hyperplanes to bifurcate them into bins. In cases where an $\mathrm{n}$ dimensional hyperplane is not sufficient, the data points are transformed to a space in which they are able to be ideally separated with hyperplanes. This is known as the "kernel trick." For an HTM SDR of n bits, an n dimensional SVM can be used [18]. This is a common method used to compare spatial pooler accuracy $[19,20]$.

\subsubsection{Union Overlap}

The union overlap method described by Ahmad and Hawkins in their paper [21] is a method specifically designed for the classification of HTM output activation patterns. The method involves first taking the union of a certain number of output patterns that are all are associated with the same class. Then, to determine if an output pattern belongs to that class, the dot product of the output vector and the union vector is taken, and, if it is greater than a threshold, the input is deemed a member of the class. The three primary benefits to this method versus an SVM for a hardware implementation are:

1. This method only requires storage of one vector per classification bucket. On die storage is limited, and off die storage has poor latency and would be a bottleneck to throughput. 
2. Only simple addition, comparison, and bitwise operations on unsigned integers is required.

3. Recomputing the union vectors can be done online whenever labels are available to adapt to a changing HTM, thus enabling a dynamic classifier suitable for an online application.

However, this method requires several assumptions be made about the HTM outputs. First, the outputs must be truly sparse [21]. Increases in output vector density translates into noise, and will cause many more bits being active in the union vector. Having a large portion of the union vector bits active will result in a higher rate of false positive matches. Second, this classification method operates under the assumption that the SDRs are distributed, as well as sparse. "Distributed" means that the activation of many neurons is necessary in order to be representative of something significant, which sets a lower limit to sparseness. So, when there is overlap between two patterns, it is highly likely that the HTM is interpreting inputs with similar features. The likelihood two random sparse vectors would have overlap is relatively small. For instance, assuming two random vectors of length 512, each with 20 bits of activation, the chance that $50 \%$ or more of the bits will overlap is $\approx 1.1 \times 10^{-10}$. If the number of active bits is increased to 50 , that probability drops even further to $\approx 8.8 \times 10^{-15}$. Of course, this assumes that the two vectors are chosen at random, but since the active columns in the spatial pooler are reinforced to be more likely to inhibit their neighbors, the output patterns of dissimilar inputs will have some correlation. Thus, the union overlap method requires some method of enforcing more entropy, or randomness between outputs corresponding to unrelated inputs. A spatial pooler exhibiting high entropy has a higher degree of homeostasis. 


\subsection{HTM Spatial Pooler Hardware Implementations}

A few efforts have been made to implement the HTM spatial pooler on reconfigurable hardware previous to this work $[7,19]$. This thesis is based primarily on the implementation described by Zyarah and Kudithipudi in their work titled "Reconfigurable Hardware Architecture of the Spatial Pooler for Hierarchical Temporal Memory" [20]. In this implementation, they introduce the concept of a "synthetic synapse". In HTM, each column is connected to many, sometimes thousands, of bits in the input vector. In hardware, having thousands of nets in the fan-in to each and every column's processing logic would be prohibitively complex from a routing perspective. Instead of having individual physical connections between the inputs of each column, the input address and permanence value for each synapse is stored in a memory element, in their case a 64-byte RAM for each column. During Phase 1 of the HTM spatial pooling algorithm, for every synapse in every column the memory holding the input data is indexed with the input space address particular to that synapse, and if that input bit is high, the synapse's permanence is compared to the threshold to compute the overlap score for each column.

Their approach capitalized on the parallel nature of the computations involved in the HTM algorithm. This method showed significant speedup of the algorithm with respect to their software simulations, a $4817 \mathrm{X}$ improvement, and using an SVM to test the classification accuracy of their model on the MNIST dataset, they achieved an accuracy of $91 \%$. Table 1 details some of the parameters used in their implementation. 
Table 1: $\quad$ Parameters used for Spatial Pooler (from Zyarah \& Dhireesha, 2015)

\begin{tabular}{|c|c|}
\hline Parameter & Value \\
\hline Number of columns & 100 \\
\hline Winning columns & $20 \%$ \\
\hline Columns per synapse & 16 \\
\hline Synapse permanence threshold & 127 \\
\hline Permanence increment/decrement factor & $+/-1$ \\
\hline Inhibition type & Global \\
\hline Minimum overlap & 2 \\
\hline
\end{tabular}

In this implementation, they chose to use a global inhibition as opposed to a local inhibition. Global inhibition works by first calculating the overlap scores of all columns, and then selecting the columns with the highest overlap, in this case the top $20 \%$, as the winners. This is in contrast to local inhibition which selects the winners by comparing the overlap score of each column to that of the columns within a certain distance, and declaring it a winner if its score is greater than or equal to the score of the columns around it. It has been shown that there is little difference between global and local inhibition when using the MNIST dataset, in terms of the feature extraction ability of the spatial pooler [22]. The minimum overlap parameter sets the minimum overlap threshold for each column. In this case, a column must have an overlap of at least 2, while also being in the top $20 \%$ of all columns to be active.

Figure 10 shows the activation patterns of two HTM regions with identical overlap scores, with the one on the left using local inhibition (radius 1, minimum overlap 
of 2), and the one on the right using global inhibition (top 6 columns declared as winners).

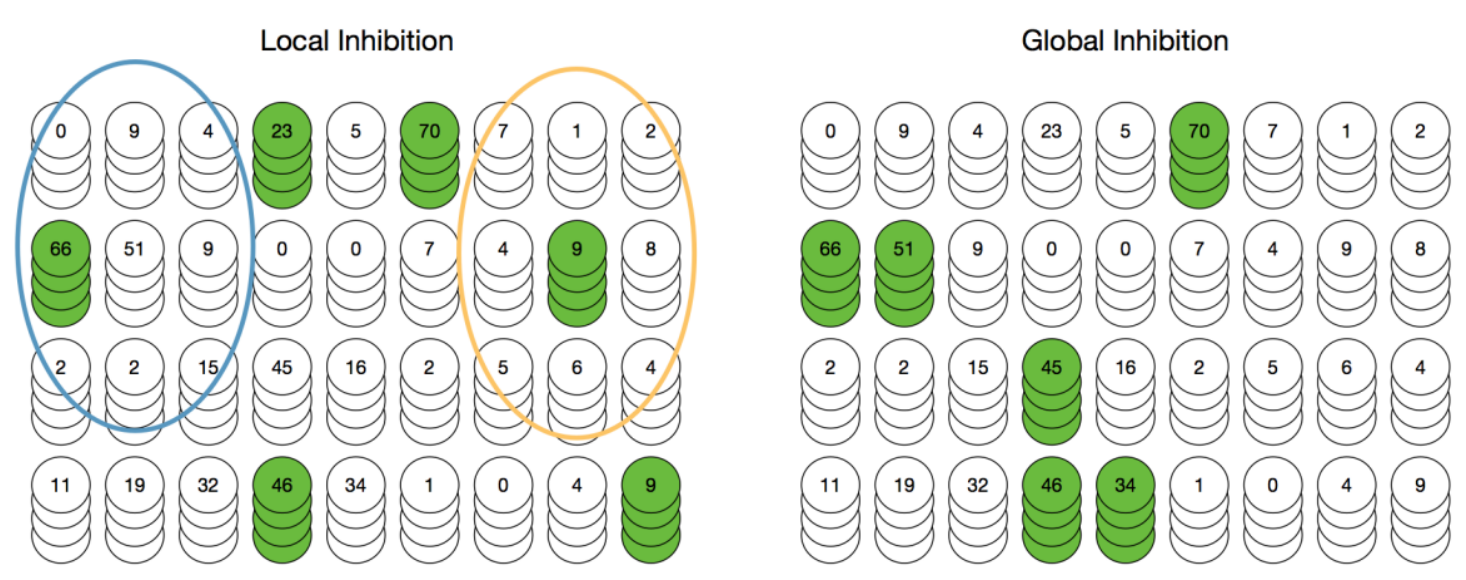

Figure 10. Inhibition comparison

These two approaches both maintain the same level of activation, but the pattern differs slightly. The blue circle in the left figure represents the inhibition radius of the column labeled "51", and the orange circle is the inhibition radius for the column labeled "9". With local inhibition, the "9" column is declared a winner since it has a higher activation than all of the columns within its radius, but is not activated with global inhibition due to its low relative overlap score. Conversely, the "51" column is inhibited with local inhibition by the "66" to its left, but is active with global inhibition since it has a high overlap relative to all other columns.

\subsubsection{Logical Implementation}

Their implementation duplicated the logic required for the overlap calculation and learning algorithm for each column in the region. Each column was comprised of LUTs, a handful of registers, and a 64-Byte RAM. Each RAM contained 16-Bytes of addresses ( 1 byte per address mapping back the input space), 16-Bytes of permanencies ( 1 byte per synapse), and 32-Bytes of input data. Each cycle the RAM would be indexed to fetch 
either the permanence of a synapse, the address of the input bit to which that synapse was connected, or the input bit itself. Thus, phase 1 of the spatial pooler was able to be computed in parallel, in relatively few cycles. The activations of the columns were fed back to the "MCU", or main control unit, via a series of shift registers. The MCU would perform a sorting algorithm on the overlap scores to determine winning columns. Then, only the winners would perform the learning phase. Figure 11 gives an overview of the logic required for each column.

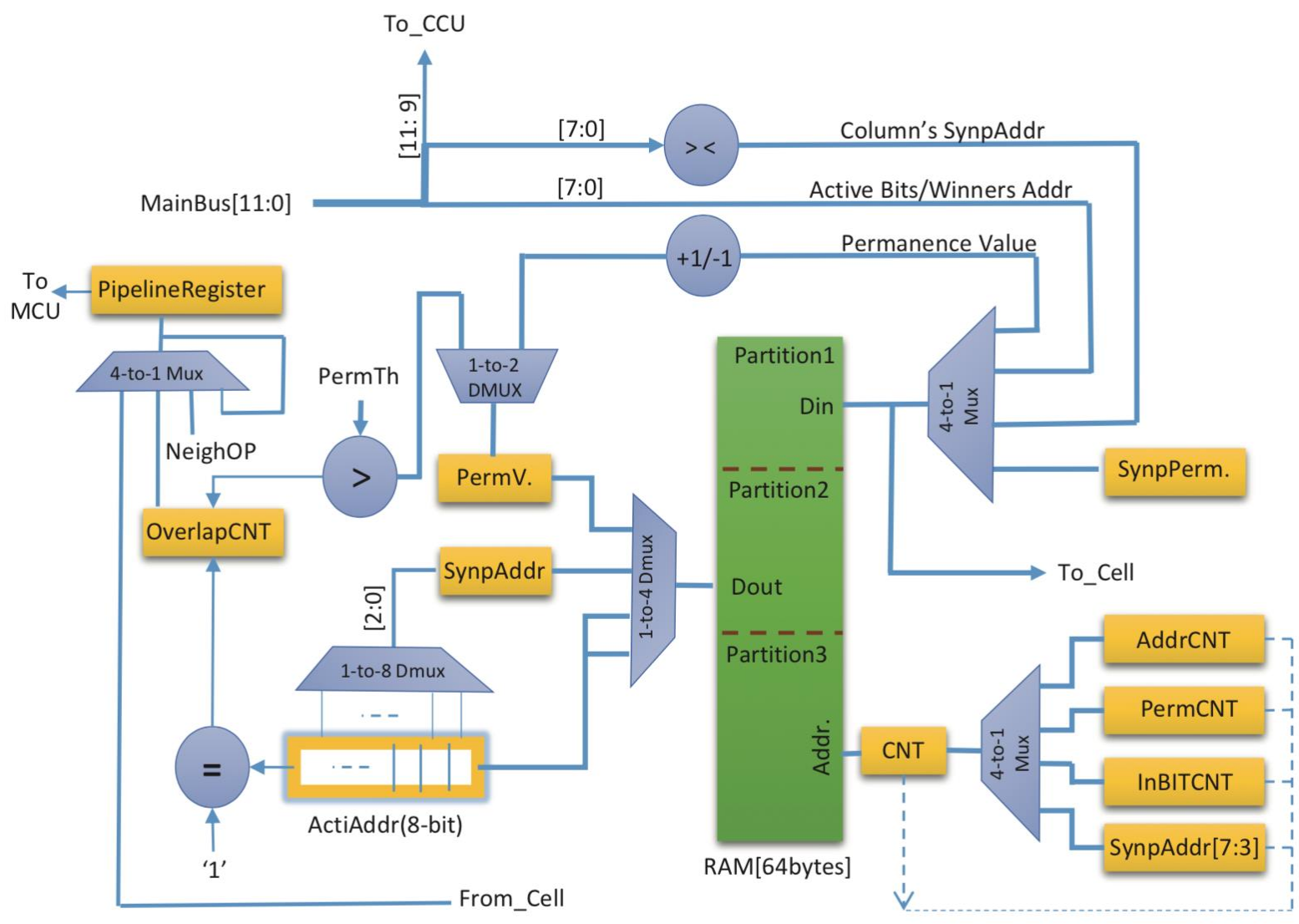

Figure 11. RTL representation of a column

Next, we discuss the drawbacks of this hardware implementation. In order to ensure every column can access the input data simultaneously in a single cycle, the entire input vector is stored in each column's RAM. This method essentially cuts in half the 
number of columns which are able to fit on the device. With 100 columns, only $1 \%$ of the space used to store the input vectors holds unique information. This metric will get worse as the complexity of the HTM increases by either increasing the number of columns, or adding regions.

Notably missing from this implementation is a method of boosting the inactive columns which is part of the core HTM spatial pooler algorithm. Failing to include the boosting mechanism would decrease the homeostasis of the HTM, and could result in reduced classification performance. When comparing the performance of HTM spatial poolers, Cui, Ahmad, \& Hawkins noted both a significant reduction in classification error rate, and increased homeostasis when boosting was used [23].

The method for classification also requires an offline process, and requires storage to preserve all of the previous activation patterns. Classification using a support vector machine necessitates storing all of the activation patterns, then processing them to build the SVM.

\subsection{MNIST Database of Handwritten Digits}

The MNIST database is a collection of 70,000 images, each containing a single numeric digit from 0 through 9. Each image is composed of 28x28, 8-bit, greyscale pixels with the digit positioned such that the center of mass is in the center of the image. The database is separated into a training set, and a classification set. 60,000 images are used for training, and 10,000 for classification. The images consist of digits written by approximately 250 writers, and each writer either provided digits for the training set or the classification set, but not for both. 
In order to reduce the size of the HTM domain, and maintain a consistent evaluation methodology with Zyarah and Kuduthipudi [20], each pixel was translated from 8-bit resolution to one bit by simply truncating the least significant 7 bits. Figure 12 shows an original MNIST digit on the left, and one converted to a 1-bit black and white scale on the right by truncating the 8-bit value to one bit.
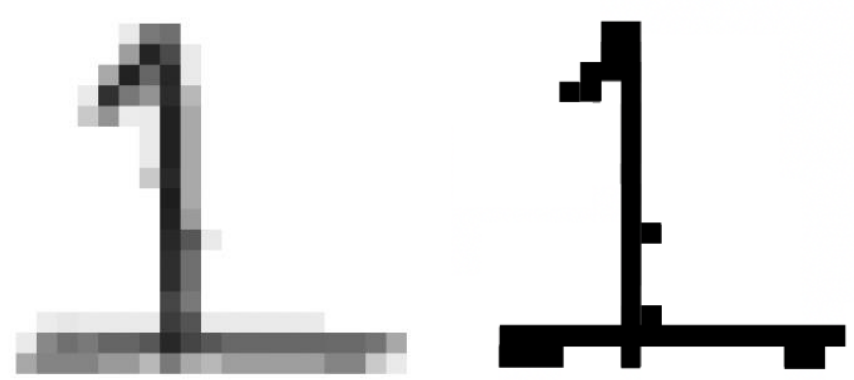

Figure 12. MNIST digit comparison 


\section{CHAPTER 3: DESIGN METHODOLOGY}

\subsection{Overview}

There are two main novel components to the hardware implementation described in this thesis: implementing the HTM boosting, and an online classification algorithm. The following sections provide details on the algorithms and their hardware implementations.

\subsection{HTM Boosting}

As previously stated, boosting is a technique used to maintain a higher level of homeostasis in column activation. Homeostasis is a property of a system to maintain some variable nearly constant across its constituent elements. In the context of the HTM, it is a measure of a region's ability to maintain similar activation duty cycles for all of its columns. An HTM with a high level of homeostasis would inhibit overactive columns, and boost less active columns to maintain a similar duty cycle across all columns. An HTM with poor homeostasis would have some columns which are exceptionally active relative to others. When a column is active across broadly differing input patterns, its activation becomes meaningless for classification, and reduces the likelihood other columns can express themselves and identify meaningful features in the input space. Boosting seeks to solve this problem by inhibiting overactive columns, boosting the less active columns, or a combination of both.

In this implementation, both boosting and inhibition are evaluated. From a high level view, boosting is achieved by keeping track of the activation duty cycles for every 
column, and increasing a columns overlap score if it is underactive, or decreasing its overlap score if it is overactive. The net effect is a more consistent use of each column in the regions activation patterns. The novel contribution of this work is implementing the HTM boosting functionality in reconfigurable hardware.

The implemented boosting algorithm computes a boosting factor for each column based on the duty cycle of that column, as well as the duty cycles of the columns within its inhibition radius. The overlap score of each column is then multiplied by this boosting factor to obtain its boosted overlap score. If the boosted overlap score of a column is higher than that of the other columns in its inhibition radius, that column is chosen as a winner for that iteration. This boosting methodology follows the boosting algorithm described in [8].

\subsection{Online Classification}

The online classification algorithm takes advantage of the fact that the output of the HTM region is an SDR. From an intuitive perspective, since the activation patterns are sparse, it is unlikely that there will be significant overlap between any two random activation patterns.

The classification method employed in this implementation is a derivative of the union overlap method. This modified method will be referred to as Scaled Union Overlap (SUO). With SUO, the union vectors are computed in the same manner, but the overlap score is then divided by the Euclidean length of each union vector. This penalizes the union vectors with longer lengths since they are more likely to have overlap with all output patterns simply because more of their elements are non-zero. 
The objective changes from finding the union vector with the highest overlap (dot product) to finding the union vector with the smallest angle between it and the pattern being tested. Take for example the situation shown in Figure 13.

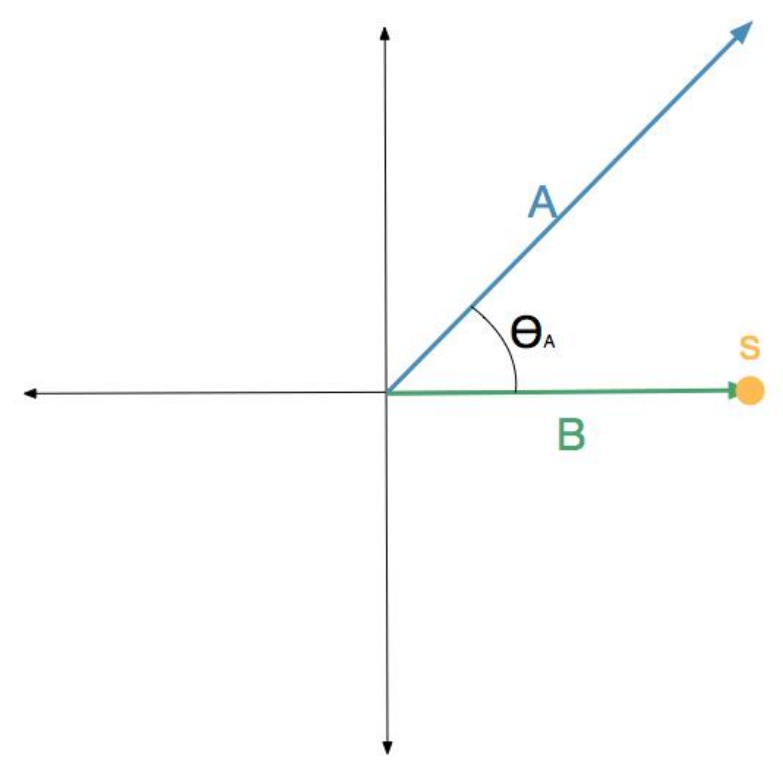

Figure 13. Scaled Union Overlap vs. regular union overlap

In this example, $\mathbf{A}$ and $\mathbf{B}$ are both union vectors representing classes $\mathbf{A}$ and $\mathbf{B}$ respectively. $\mathbf{s}$ is a vector to be classified as either class A, or class B. For the purposes of this example, assume $\mathbf{A}, \mathbf{B}$, and $\mathbf{s}$ are defined as follow:

$$
\boldsymbol{A}=(1,1), \boldsymbol{B}=(1,0), \boldsymbol{s}=(1,0)
$$

In the union overlap method, both $\boldsymbol{s} \cdot \boldsymbol{A}$ and $\boldsymbol{s} \cdot \boldsymbol{B}$ are both 1 . However, visual inspection shows that $\mathbf{s}$ is more similar to $\mathbf{B}$ than to $\mathbf{A}$. In scaled union overlap, the dot products are divided by the length of the union vector to obtain $|\boldsymbol{s}| \cos \left(\theta_{u}\right)$. For all union vectors, $|\boldsymbol{s}|$ remains constant, and all elements of each vector are non-negative, so finding $\mathbf{U}$ which satisfies $\max \left(|\boldsymbol{s}| \cos \left(\theta_{u}\right)\right), \forall \boldsymbol{U}$ is the same as finding $\min \left(\theta_{u}\right), \forall \boldsymbol{U}$. The method for finding the best $\boldsymbol{U}_{\boldsymbol{n}}$ vector then becomes simply iterating through all $\boldsymbol{U}_{\boldsymbol{n}}$, and keeping track of the best $\mathbf{U}$ which satisfies inequality 9 . 
$\frac{s \cdot U_{n}}{\left|U_{n}\right|}>\frac{s \cdot U_{\text {best }}}{\left|U_{\text {best }}\right|}$

The algorithm can be summarized with the pseudo-code shown in Figure 14.

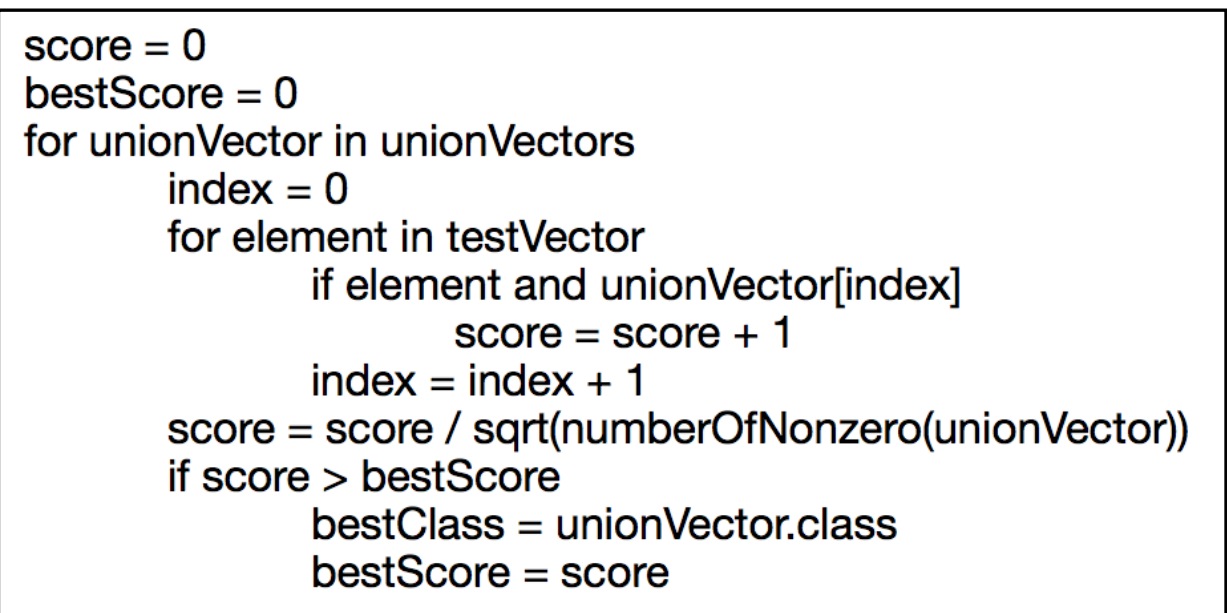

Figure 14. Scaled Union Overlap pseudo-code

For each union vector, the dot product is computed, then divided by the length of the union vector. Since the union vectors are bit strings, the Euclidean length of the vector is simply the square root of the sum of all of the non-zero elements. Making this substitution in inequality 9 yields inequality 10 .

$$
\frac{s \cdot U_{n}}{\sqrt{\operatorname{sum}\left(U_{n}\right)}}>\frac{s \cdot U_{\text {best }}}{\sqrt{\operatorname{sum}\left(U_{\text {best }}\right)}}
$$

In order to preserve the precision of the calculations and avoid floating point representations, the hardware implementation modifies this comparison slightly. Inequality 10 is altered to become inequalities 11 , then 12 .

$$
\begin{aligned}
& \frac{\left(s \cdot U_{n}\right)^{2}}{\operatorname{sum}\left(U_{n}\right)}>\frac{\left(s \cdot U_{\text {best }}\right)^{2}}{\operatorname{sum}\left(U_{\text {best }}\right)} \\
& \left(s \cdot U_{n}\right)^{2} \times \operatorname{sum}\left(U_{\text {best }}\right) \\
& \quad>\left(s \cdot U_{\text {best }}\right)^{2} \times \operatorname{sum}\left(U_{n}\right)
\end{aligned}
$$

Doing this comparison avoids taking square roots and dividing, both of which are computationally expensive, and allows the use of only integer arithmetic which avoids 
any loss of precision. Including this modification produces the pseudo-code shown in Figure 15.

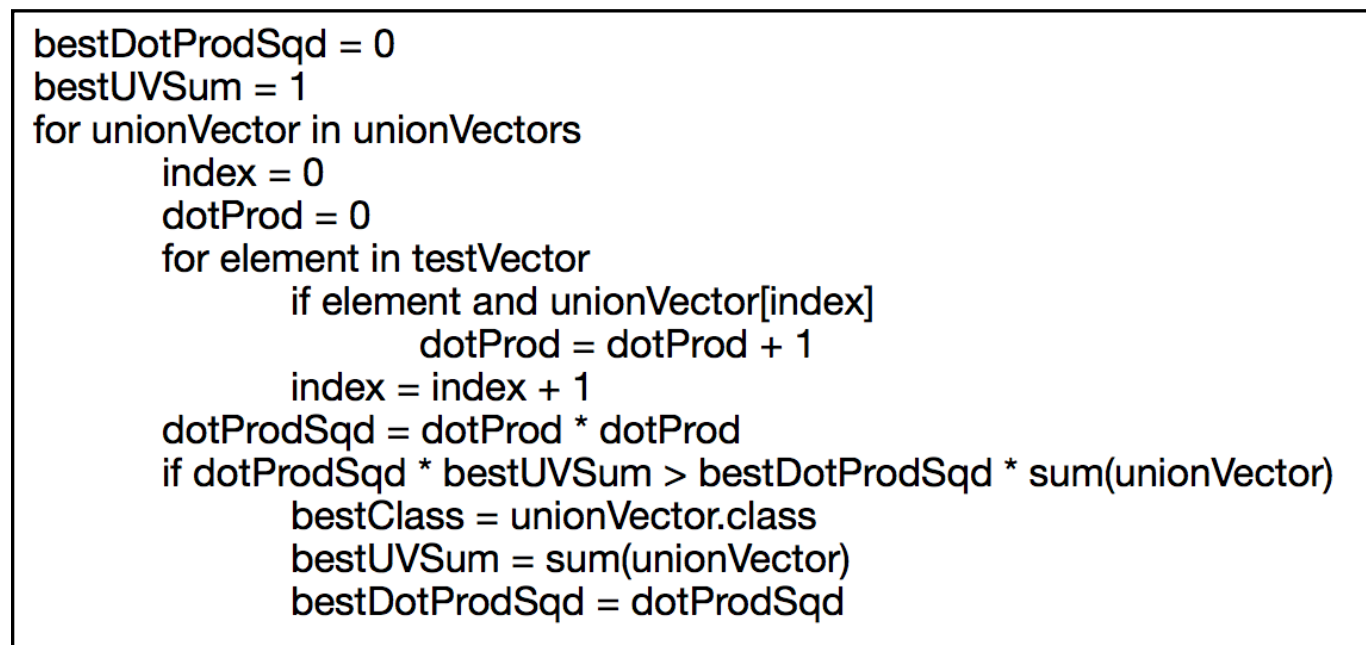

Figure 15. Modified scaled union overlap pseudo-code

Making such modifications removes the need for a DSP block to be implemented in the FPGA to perform the division or square root functions, and if vectors of sufficient size are used, there is no loss of precision due to using a fixed width floating-point representation. 


\section{CHAPTER 4: HARDWARE IMPLEMENTATION}

\subsection{Overview}

The RTL design for the FPGA implementation was written in Verilog using the 2001 standard, and was synthesized for and tested with a Digilent Zybo board containing a Xilinx Zynq XC7Z010 programmable SoC [24]. The HTM core was packaged as an AXI peripheral and communicated instructions and data on that bus with the on-die hardcore ARM based processor. A compiled C program was run on the processor to retrieve the test and classification vectors, along with their associated labels, and relay them to the HTM core (htm_core). The interface between htm_core and the AXI bus consisted of:

1. A set of control registers

2. An SRAM for transferring input vectors to the htm_core, and delivering the activation patterns back to the processor

The htm_core performs the spatial pooling algorithm, and either trains or exercises the classifier based on the status of a configuration register. First, the training vectors are loaded, and then the classification vectors. During classification, the processor compares the classifications returned by the htm_core with the correct classes read from a label file located on a micro-SD card. It then communicates the results back to a terminal connected through UART. The output vectors, or activation patterns, are also sent back to the processor via AXI, and where they can be written to a file on the micro-SD storage card. This information is then transferred to a more powerful machine for SVM classification (see Appendix A for specifications). 
The htm_core is made of several distinct components:

1. A Finite State Machine (FSM) to control all of the constituent parts of the htm_core

2. Linear Feedback Shift Register (LFSR) used for initializing the synapses to psuedorandom values

3. Set of SRAMs used to store the synthetic synapses

4. A second FSM to select the winning columns based on the activations of the columns within an inhibition radius

5. An SRAM to store the duty cycles of each column

6. A second SRAM used to store the union vectors required for classification

The detailed RTL description of each of these components are described in the following sections.

\subsection{Primary Finite State Machine}

Two state machines are employed to manage the control signals which are detailed in the other RTL modules. The primary FSM is responsible for several functions:

1. Initialization of the synapses

2. Communication with the AXI RAM

3. Interfacing with the synapse RAMs

4. Controlling the training and classification functions

5. Keeping track of the duty cycles of the columns

Figure 16 illustrates the states and their relationships. 


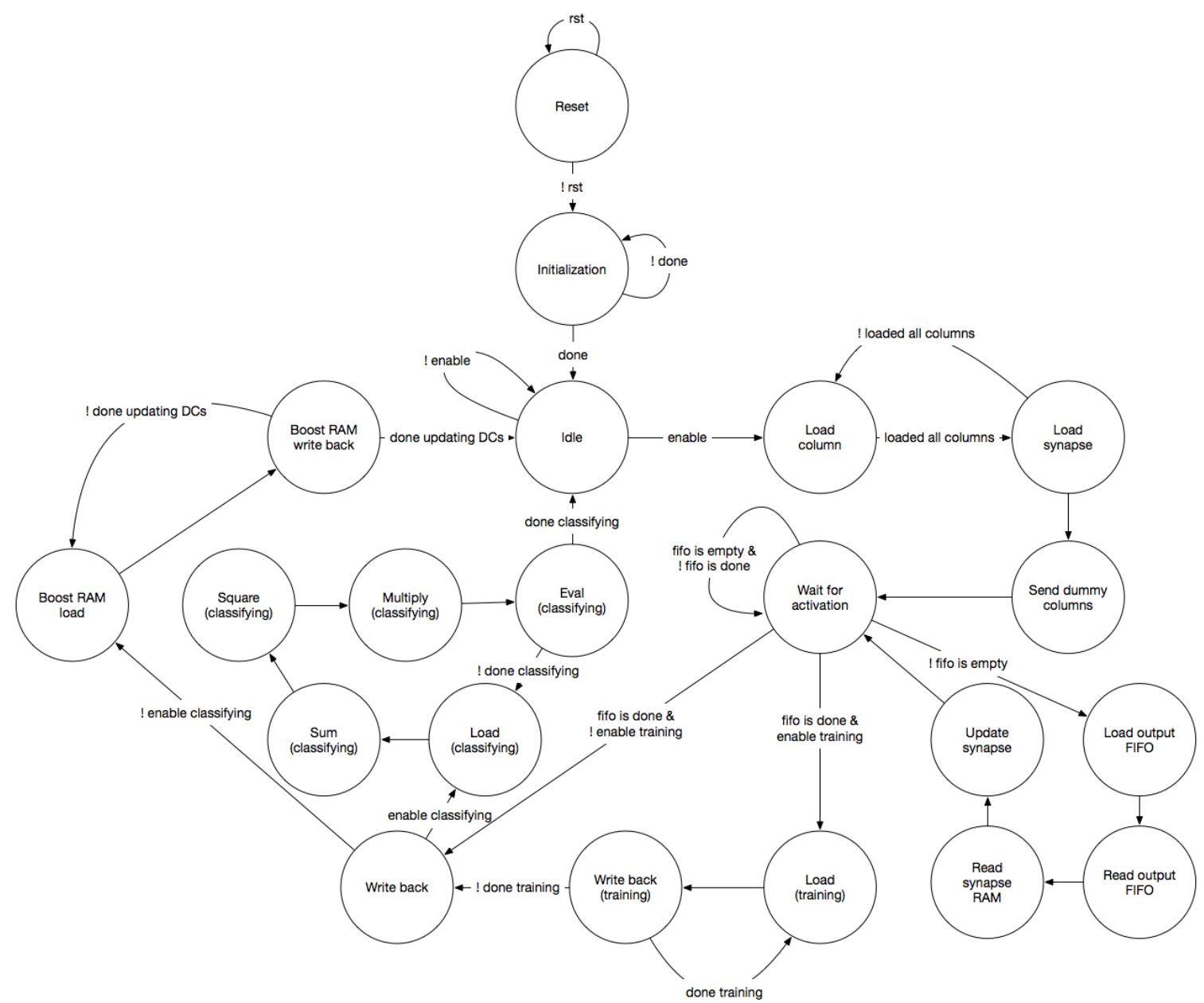

Figure 16. Primary FSM diagram

The initial state is the "Reset" state, where all registers are reset to their initial values. During "Initialization" the LFSR outputs are written to the synapse RAM to their pseudo-random values. The FSM stays in the "Idle" state until the enable is asserted. Once asserted, the addresses and permanencies are read from the synapse RAM one column at a time. Using these addresses, the input vector values are read from the AXI interface RAM one address at a time. When all data for a column have been read, that data is pushed to the "input FIFO" for the second FSM to process. After all columns have been fed to the second FSM, a number of "dummy" columns are sent to flush out the input FIFO. As the secondary FSM processes the columns, the primary FSM idles in the 
"Wait for activation" state. As data is returned through the "output FIFO", the "fifo is empty" signal is asserted, and the synapse permanencies are updated. Once all winning columns have been updated, if training is enabled, the union vectors are updated. Otherwise the "Write back" phase begins. During "Write back" the activation vector is deposited to the AXI interface RAM. If classification is enabled, the union vector RAM is read, and the class with the largest scaled union overlap value is selected as the winner. If classification is not enabled, then the duty cycles are updated instead of performing classification. Once the algorithm has completed, the FSM returns to the "Idle" state, and awaits further input.

\subsection{Linear Feedback Shift Register}

A linear feedback shift register (LFSR) is a common tool used to implement a pseudo-random number generator. This LFSR is 128 bits long, with XOR taps at bits (from 1 to 128 ) 128, 126, 101, and 99. This gives a unique sequence of $2^{128}-1$ bit strings of length 128 until the sequence repeats [25]. The LFSR is used to initialize the HTM synapses with pseudo-random starting permanencies, and mappings to the input space.

If desired, the htm_core can be configured to restrict the mapping of the columns to a particular range of bits within the input space, and is controlled via a compiler macro. The motivation for restricting the input range in this way comes from a suggestion made by Mnatzaganian, Fokoue \& Kudithipudi [22]. Their work involved characterizing the HTM spatial pooler using the MNIST dataset, and suggested the spatial nature of image data could be exploited by restricting the input of each column to a cluster of nearby pixels. 
This implementation uses a span width of 112 bits, which corresponds to 2 rows of pixels in an MNIST dataset image. Each consecutive column will shift its valid window by 2 bits. The first column would have possible connections to inputs 0 through 111 , then the next column could be connected to 2 through 113, and so on. Figure 17 illustrates this concept of "spanning". In the example, each column can have a span of 6 with the increment is set to 2 .

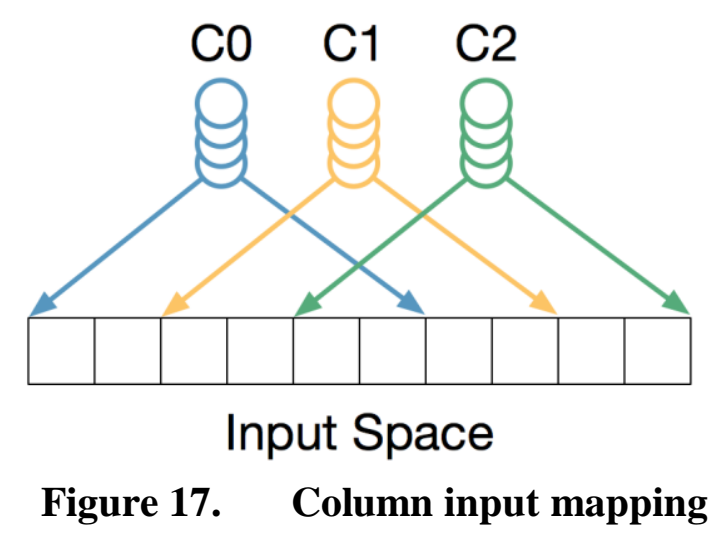

If the number of columns multiplied by the increment value plus the width is greater than the number of utilized inputs, as is the case with this particular use case, the upper and lower address limits must be adjusted when they extend beyond the number of utilized inputs. For example, see Figure 18, where LB, UB, and IU represent the lower bound, upper bound, and number of inputs used respectively. 


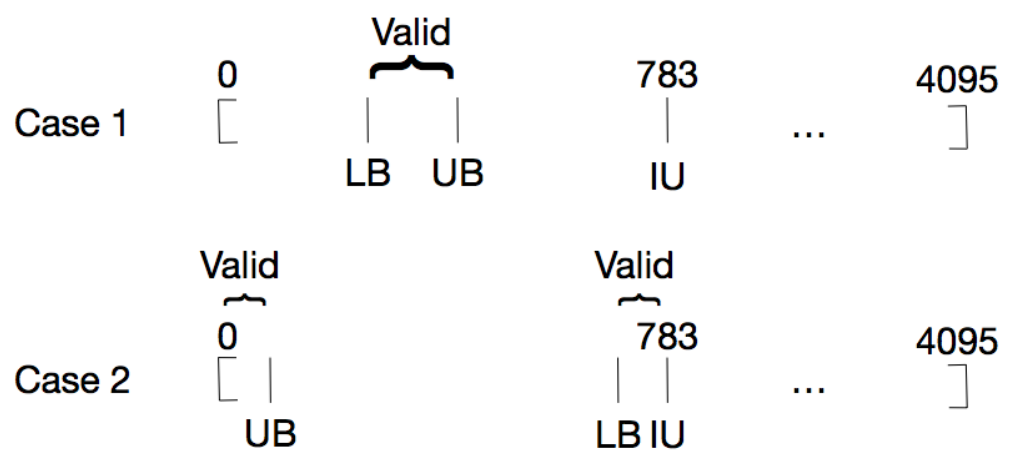

Figure 18. Valid rang of input bits

As the figure illustrates, there are three valid ranges for the two different cases.

Figure 19 is a block diagram of the LFSR and shows how its output bits are used to generate the initial synapse values.

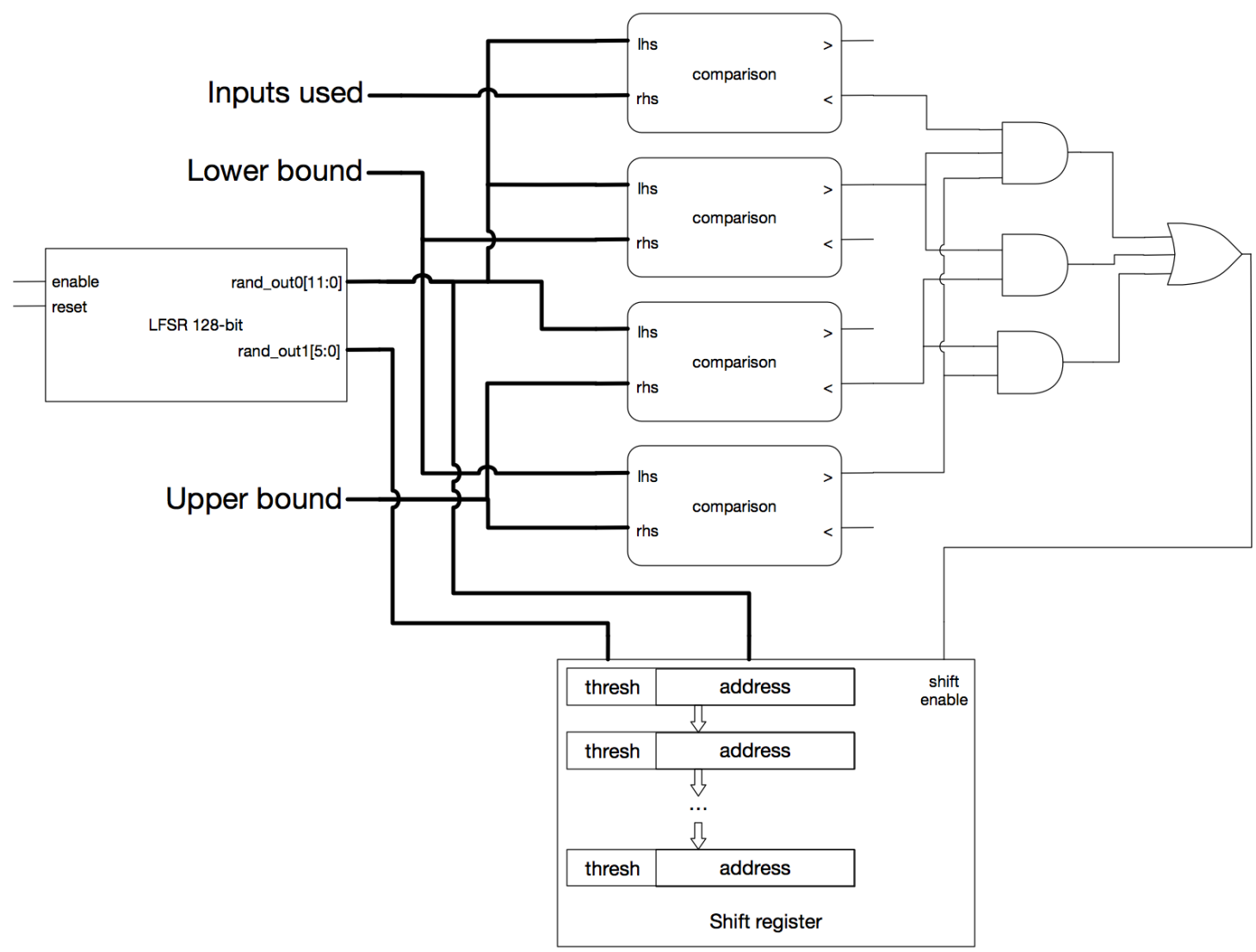

Figure 19. Linear feedback shift register diagram 
For every column in the SP, the shift register is filled with 48 synapses worth of addresses and thresholds. The permanencies are initialized to values between 28 and 35 by adding 28 to a pseudo-random 3-bit number. Once the shift register has been filled with the 48 valid synapses, they are written to the synapse SRAMs for storage. The htm_core initialization is complete once all 512 columns have received initial values.

\subsection{Synapse RAMs}

In this design, several 36KB single port SRAMs are used to store the synapse data. They are arranged in parallel with read/write widths of 72 bits each, and have a depth of 512 words. Each synapse is composed of 12 address bits to map to the input space, and 6 bits of permanence. Utilizing 12 SRAMs with 72 bits of input data, 48 synapses, each consisting of 18 bits, can be written to or read from the SRAM array in a single cycle. Access to these RAMs is controlled by the primary state machine, which initializes its values, reads the data, and updates permanencies during SP phase 3.

Reading of the synapse RAM is done once per column. The addresses and permanencies are used to index the input SRAM, and to determine whether each synapse is connected or unconnected by comparing the permanence to the threshold. This data is sent to the "winner selection state machine" through the "input FIFO". After winners have been selected, permanence values are incremented or decremented based on the state of the input bit associated with each synapse. Figure 20 illustrates this structure. 


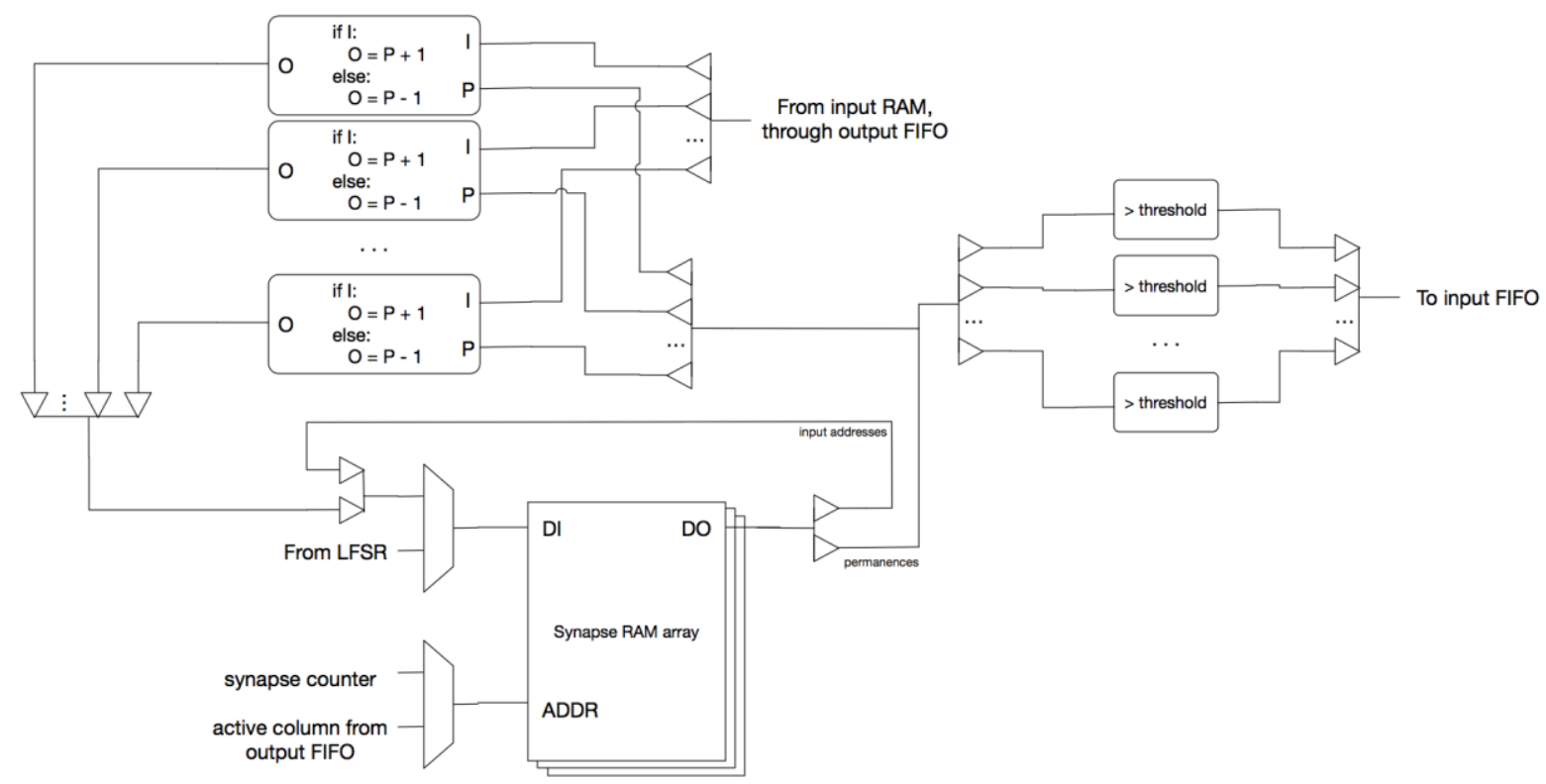

Figure 20. Synapse RAM

\subsection{Winner Selection State Machine}

The process of selecting winning columns in the spatial pooler is performed by a second independent finite state machine (FSM) which communicates with the primary state machine via two SRAMs operating as FIFOs: an input FIFO, and an output FIFO. The states and their relationships are shown in Figure 21. 


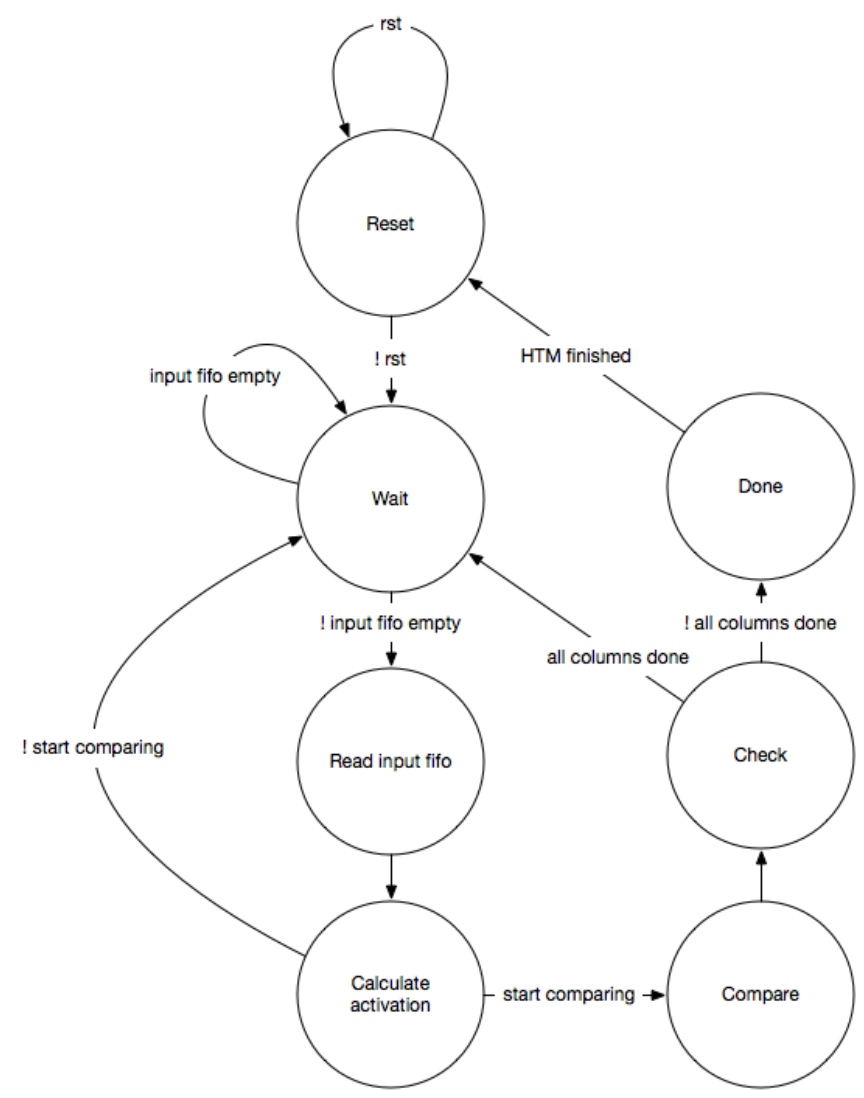

Figure 21. Winner selection SM diagram

The primary FSM retrieves the input from the input SRAM one bit at a time, utilizing 2 clock cycles per synapse per column. So, every 96 clocks $(48 \cdot 2)$ the primary FSM collects all of the data necessary for an entire column. The primary FSM passes to the winner selection FSM four values:

1. The moving average duty cycle of the column (11-bits),

2. The largest duty cycle of all columns in the current column's inhibition radius (11bits),

3. 48-bits representing whether each synapse is connected vs unconnected, and 4. 48-bits representing the inputs associated with each synapse. 
As the FIFO receives data from the primary FSM, the winner selection FSM will read the first entry in the FIFO, process it, and push the results into a shift register. The FSM first uses the input space mapping and connectedness information from each synapse to compute the overlap for the column. Then, the duty cycle of the column, as well as the largest duty cycle of all columns in the inhibition radius, is used to compute a "boosted overlap" value. This boosted overlap and the input pattern are pushed into the shift register for processing. The depth of the shift register is $2 \times$ inhibition radius +1 , in this case 21. As the values for each column fill the shift register, the boosted activation of the center column is compared against the boosted activations of each of the columns in the inhibition radius to determine if that column is deemed a winner. A column is declared a winner if its boosted activation is greater than both the minimum activation, as well as the boosted activations of 19 out of the 20 columns in its inhibition radius. Assuming a minimum activation of 1 , this constraint still guarantees an activation pattern density of at most $1 / 6$, or approximately $16.7 \%$.

For each column, a boosting factor is calculated based on the duty cycle of each column as measured over the last 2048 iterations. The column's activation is then multiplied by its boosting factor to obtain the boosted activation. If the duty cycle is above a certain threshold, then a unity boost factor is used. Otherwise, the boost factor for $b_{i}$ for the column is a linear function of the duty cycle $D_{i}$ of the column, the minimum duty cycle $m_{i}$, and the max boosting factor B as seen in Equation 13. Equation 14 shows how the minimum duty cycle $m_{i}$ is calculated from the maximum duty cycle of all columns in the inhibition radius $M_{i}$, and a non-negative integer s. If the duty cycle for the 
column is greater than the computed minimum, then a unity boosting factor (no boosting) is used.

$$
\begin{aligned}
b_{i} & =\left\{\begin{aligned}
1, & D_{i}>m_{i} \\
B+D_{i} \times \frac{(1-B)}{m_{i}}, & D_{i} \leq m_{i}
\end{aligned}\right. \\
m_{i} & =\frac{M_{i}}{2^{s}}
\end{aligned}
$$

Since the minimum duty cycle $m_{i}$ is a non-constant registered value, the boost factor calculation in Equation 13 is altered to be scaled by $m_{i}$ in order to avoid the division operation. Thus a new boosting factor $\widehat{b_{l}}$ is computed as shown in Equation 15 .

$$
\widehat{b_{l}}=b_{i} \times m=\left\{\begin{array}{rc}
m_{i}, & D_{i}>m_{i} \\
B \times m_{i}+D_{i} \times(1-B), & D_{i} \leq m_{i}
\end{array}\right.
$$

Figure 22 details the logic used to track the duty cycle of each column. A single SRAM is used to store the duty cycles. The duty cycle logic keeps track of the number of times each column is activated during a specific interval. Every $2^{11}$ iterations of the spatial pooler, the running sum of activation counts are latched, and the running sums are reset to 0 . The latched duty cycle counts are the $D_{i}$ values used to calculate the boosting factors for each column $\widehat{b_{l}}$ in equation 15 . 


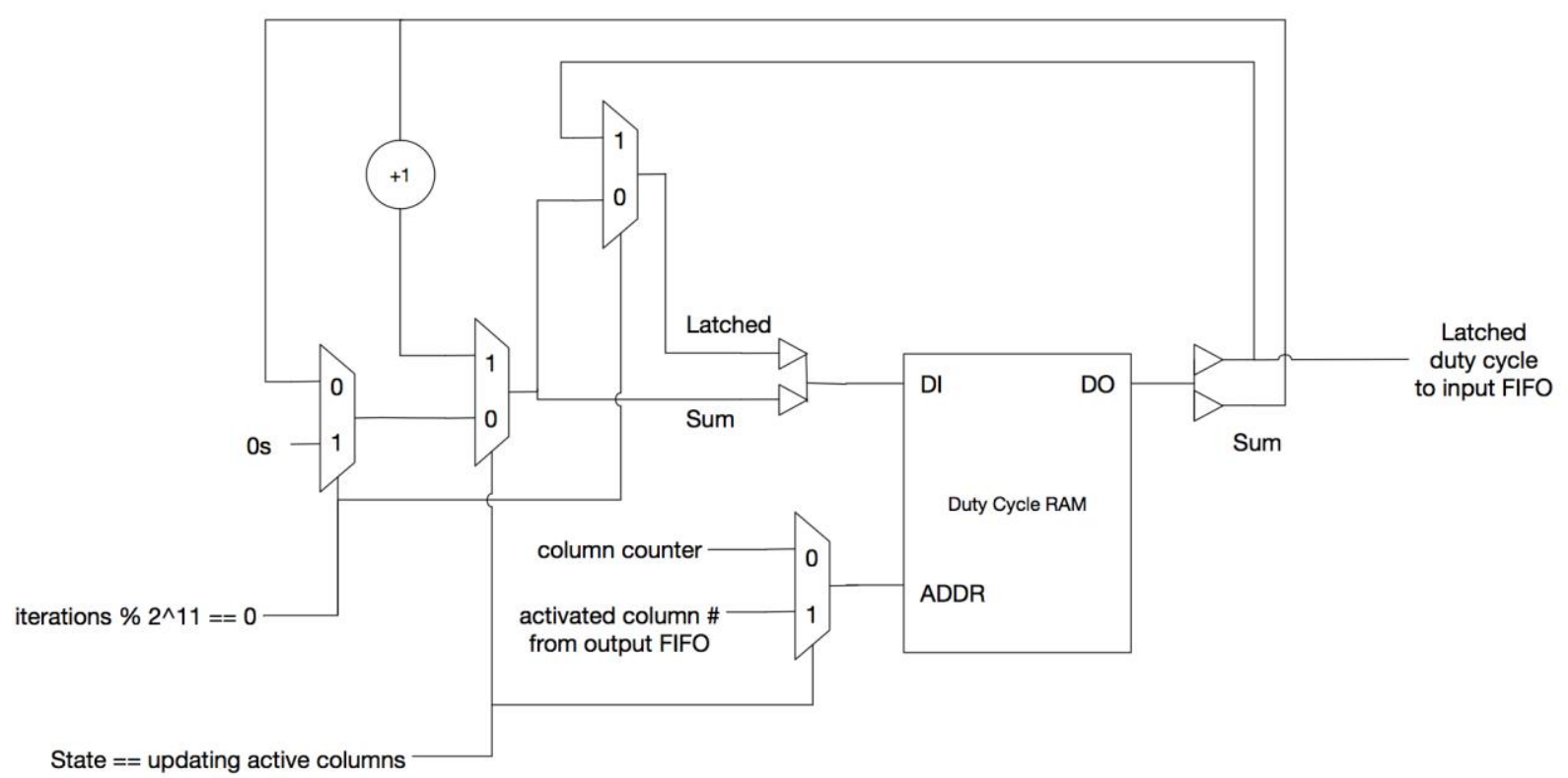

Figure 22. Duty cycle computation

Figure 23 demonstrates the logic necessary to compute the boosted activation.

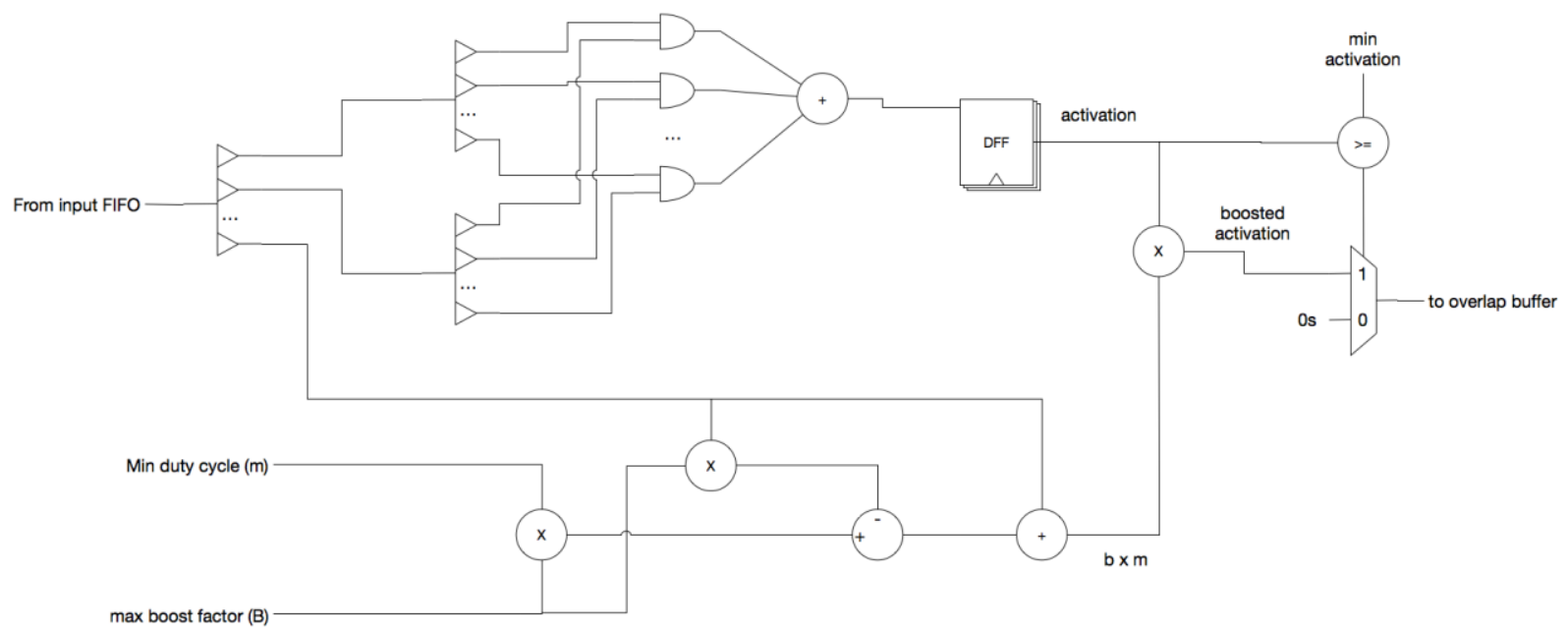

Figure 23. Boosting logic

\subsection{Classification Logic}

The final major logic component is the scaled union overlap classifier. The classifier is comprised of an SRAM used to store the union vectors, logic used to update 
the union vectors during training, and logic to compare an output vector with the union vectors in order to select the winning class.

The union vector SRAM DI and DO busses are composed of 3 sections:

1. A segment of the running union vector (16 bits)

2. A segment of the "latched" union vector (16 bits)

3. The number of active bits in the "latched" union vector segment ( 5 bits)

Utilizing one $36 \mathrm{~Kb}$ SRAM, union vectors can be stored for up to 31 distinct classes.

Once the activation pattern is computed during training, the tag provided for the input vector is used to compute the address of the first section in the union vector RAM for that particular tag. The current activation pattern is OR'ed bitwise with the union vector in 16 bit increments, until all 512 bits of the activation pattern have been utilized. Every 100 times a tag is trained, the union vector is "latched". Then the sum of active bits are also computed and stored. When classification begins, only the latched union vectors are used in the scaled union overlap classification phase. This ensures that each union vector is comprised of the same number of output vectors.

During classification, writing to the RAM is disabled, and registers containing the best tag (best_tag), the number of active bits of the union vector corresponding to the best tag (best_length), and the squared dot product of the output activation and the same union vector (best_overlap_squared) are all reset to their initial values as given in Table 2 . 
Table 2. Classification registers initial values

\begin{tabular}{|c|c|}
\hline Register & Initial value \\
\hline best_tag & 5'd0 \\
\hline best_length & 9'd1 \\
\hline best_overlap_squared & 0 \\
\hline
\end{tabular}

The dot product of the output and the latched union vector representing class "0" is computed at a rate of 16 bits per clock cycle. Once complete, the comparison described in equation 12 is evaluated to determine if class " 0 " is a better match than the tag contained in "best_tag". This process is performed until all union vectors have been tested, and the remaining "best_tag" register then holds the resulting class. The comparison operation utilizes three clock cycles. Squaring of the dot product comes first, multiplication second, with the comparison operation occurring on the third clock cycle. Squaring of the dot product is performed with a ROM. The number to be squared is used as the address bus to the ROM, and the output is the precomputed squared value contained at the corresponding address. Figures 24 shows the union vector SRAM implementation while the classification logic implementation is shown in Figure 25. 


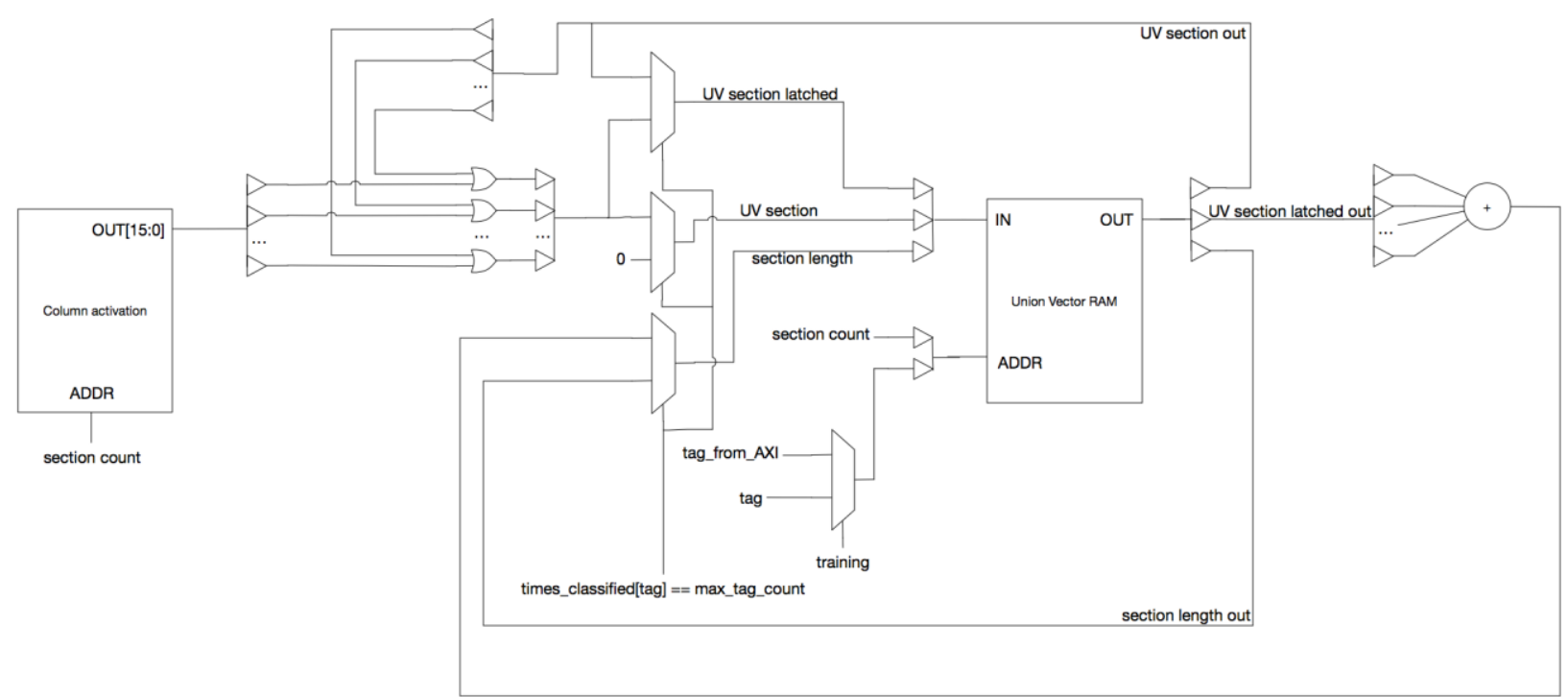

Figure 24. Union vector SRAM

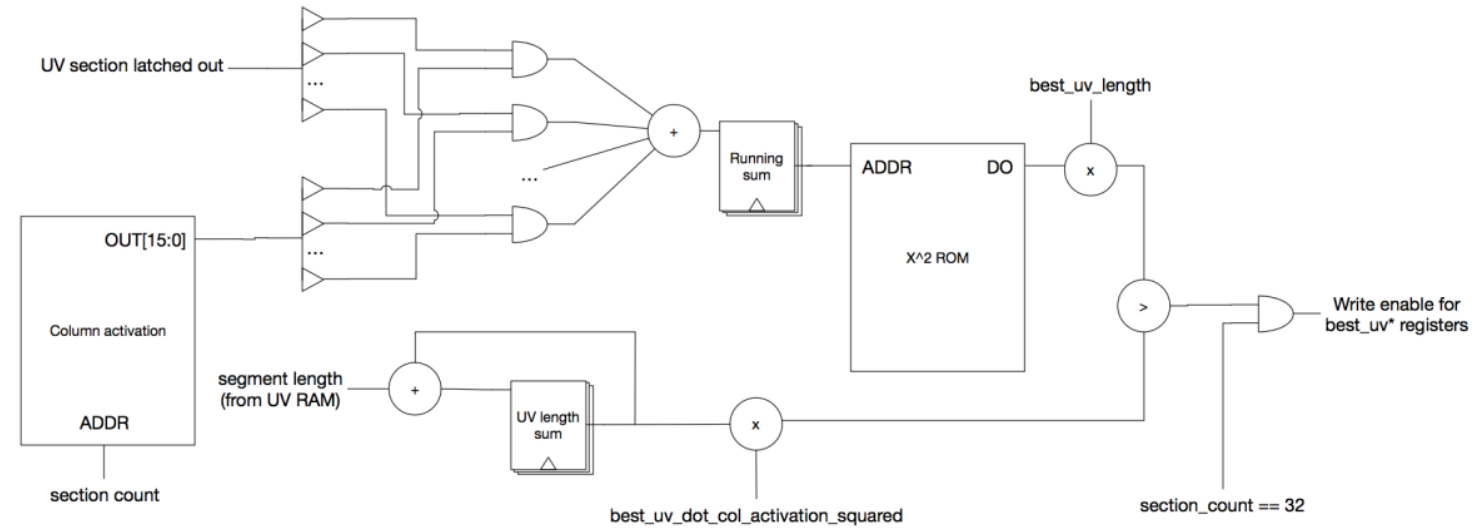

Figure 25. Classification logic 


\section{CHAPTER 5: RESULTS}

\subsection{Overview}

This chapter documents the results of both the software and hardware implementations of both the spatial pooler and the scaled union overlap classification method. Following these sections, a final summary is presented, along with some suggestions for future work.

\subsection{Software}

The Python implementation of the spatial pooling algorithm is used to sweep across several parameters for the spatial pooler to identify the optimal topology, as well as evaluate deviations from the canonical spatial pooling algorithm. The deviations from the spatial pooling algorithm produced only marginally better classification results, so were discarded in favor of evaluating only the implementation of the SP itself, and not the alterations.

As explained earlier, several parameters were swept with the Python script to determine the following optimal settings:

1. Number of columns

2. Number of synapses per column

3. Permanence threshold

4. Inhibition radius

5. Minimum target active columns (used for variable minimum overlap)

6. Maximum target active columns (used in for variable minimum overlap) 
7. Minimum overlap

8. Maximum boost factor

9. Span (the size of the subsection of the input space each column can address)

The method to test each parameter was to use a common set of settings as a baseline, and vary the only the parameter being tested while leaving all others static. The metrics used for the tests were the classification accuracy of an SVM and the scaled union overlap method. An underlying, and likely incorrect, assumption was that each of these parameters were independent variables with respect to the classification accuracy metrics. In all cases, five tests were conducted for each experiment, each with a unique RNG seed to initialize the spatial pooler's synapses. The average classification accuracy and standard deviations of the five tests are being reported in the following subsections. These metrics are reported for both the SVM, and scaled union overlap classification methods. In each table, the baseline results are highlighted in blue. Table 3 summarizes the baseline settings, as well as the values tested. 
Table 3. Software parameter sweep

\begin{tabular}{|c|c|c|}
\hline Parameter & Baseline value & Tested values \\
\hline Columns & 512 & 256,512 \\
\hline Synapses per column & 32 & $16,32,48$ \\
\hline Permanence threshold & 30 & $16,20,24,26,28,30,32$ \\
\hline Inhibition radius & 4 & $2,4,10,20$ \\
\hline $\begin{array}{c}\text { Minimum target activated } \\
\text { columns }\end{array}$ & Unused & $0,25,50,75,100$ \\
\hline $\begin{array}{c}\text { Maximum target activated } \\
\text { columns }\end{array}$ & Unused & $25,50,75,100$, Number of columns \\
\hline Maximum boost factor & 1 (no boost) & $1,1.5,2,3$ \\
\hline Minumum overlap & 1 & $28,56,84,5,6,7,8,9,112,140,168$, Input size \\
\hline Span & Input size (no span) & \\
\hline
\end{tabular}

\subsubsection{Number of Columns}

The SP was tested with two different numbers of columns, 256, and 512. In the case of scaled union overlap, increasing the number of columns provided a slight bump to classification accuracy. For this reason, 512 columns will be used in the hardware implementation. The results are shown in Table 4. 
Table 4. Number of columns software sweep

\begin{tabular}{|c|c|c|c|c|}
\hline Columns & SVM Mean \% & SVM STD & SUO Mean \% & SUO STD \\
\hline 256 & 92.61 & 0.51 & 41.08 & 2.41 \\
\hline 512 & 92.91 & 0.33 & 44.56 & 2.74 \\
\hline
\end{tabular}

\subsubsection{Synapses per Column}

Interestingly, the trend seems to be opposite for the SVM and scaled union overlap accuracies when varying the number of synapses per column. However, each of the scaled union overlap means are within two standard deviations of each other. For the hardware implementation, 48 synapses per column will be used. Results are shown in Table 5.

Table 5. Synapses per column software sweep

\begin{tabular}{|c|c|c|c|c|}
\hline Synapses per column & SVM Mean \% & SVM STD & SUO Mean \% & SUO STD \\
\hline 16 & 93.66 & 0.15 & 43.45 & 2.13 \\
\hline 32 & 92.91 & 0.33 & 44.56 & 2.74 \\
\hline 48 & 92.87 & 0.21 & 45.98 & 1.85 \\
\hline
\end{tabular}

\subsubsection{Permanence Threshold}

Setting a lower threshold seems to correlate with an increase in classification accuracy. One possible explanation for this is that when the threshold is higher, there may be more columns which are initialized as "dead" from the beginning. The synapses from these columns may have low initial permanence values, and are unable to ever achieve a high enough activation to participate in learning. When the threshold is lower, it reduces the bias towards selecting columns with synapses with high initial permanencies making it possible for the columns with the most significant proximal connections to stand out 
among the others. Table 6 includes an additional column showing the entropy calculated for the activation patterns for the different configurations. Permanence threshold seems to also be negatively correlated with entropy, showing that reducing the similarity between activation patterns correlates to better classification.

Table 6. Permanence threshold software sweep

\begin{tabular}{|c|c|c|c|c|c|}
\hline Threshold & SVM Mean \% & SVM STD & SUO Mean \% & SUO STD & Entropy \\
\hline 16 & 93.66 & 0.15 & 49.31 & 1.37 & 421.82 \\
\hline 20 & 92.22 & 0.12 & 47.63 & 2.31 & 428.34 \\
\hline 24 & 93.13 & 0.21 & 50.54 & 1.42 & 430.78 \\
\hline 26 & 93.16 & 0.21 & 46.87 & 3.23 & 427.83 \\
\hline 28 & 93.01 & 0.36 & 46.48 & 2.65 & 424.00 \\
\hline 30 & 92.91 & 0.33 & 44.56 & 2.74 & 417.13 \\
\hline 32 & 92.42 & 0.48 & 42.10 & 2.30 & 406.51 \\
\hline
\end{tabular}

\subsubsection{Inhibition Radius}

As shown by Table 7, increasing the inhibition radius of the columns also increases classification accuracy, as well as decreases variation. However, increasing the inhibition radius also increases runtime. The hardware will use an inhibition radius of 10 columns. 
Table 7. Inhibition radius software sweep

\begin{tabular}{|c|c|c|c|c|}
\hline Inhibition radius & SVM Mean \% & SVM STD & SUO Mean \% & SUO STD \\
\hline 2 & 92.41 & 2.02 & 47.42 & 14.77 \\
\hline 4 & 92.91 & 0.33 & 44.56 & 2.74 \\
\hline 10 & 94.82 & 0.26 & 54.73 & 1.22 \\
\hline 20 & 94.33 & 0.12 & 61.29 & 1.59 \\
\hline
\end{tabular}

\subsubsection{Variable Minimum Overlap}

Having a variable minimum overlap was an enhancement explored during the software experiments. The HTM was presented with both minimum and maximum targets for activated columns during each iteration. During training, if the number of active columns was outside the target range the minimum overlap would either be raised or lowered by one to stay within the desired range. The effect was an integral feedback system to control the number of active columns. The results of this experiment, shown in Table 8, were promising, but interfered with the boosting mechanism when implemented together. 
Table 8. Variable min overlap software sweep

\begin{tabular}{|c|c|c|c|c|c|}
\hline $\begin{array}{c}\text { Minimum } \\
\text { columns }\end{array}$ & $\begin{array}{c}\text { Maximum } \\
\text { columns }\end{array}$ & $\begin{array}{c}\text { SVM Mean } \\
\%\end{array}$ & $\begin{array}{c}\text { SVM } \\
\text { STD }\end{array}$ & SUO Mean \% & SUO STD \\
\hline No minimum & No maximum & 92.91 & 0.33 & 44.56 & 2.74 \\
\hline 12 & 25 & 87.06 & 2.00 & 31.02 & 27.40 \\
\hline 25 & 50 & 90.51 & 0.93 & 31.78 & 27.98 \\
\hline 37 & 75 & 92.52 & 0.24 & 58.72 & 10.29 \\
\hline 50 & 100 & 92.79 & 0.37 & 44.58 & 23.88 \\
\hline 75 & 150 & 94.48 & 0.07 & 54.00 & 1.75 \\
\hline 100 & 200 & 93.00 & 0.29 & 45.00 & 1.42 \\
\hline
\end{tabular}

\subsubsection{Boosting}

Increasing the maximum boosting factor shows a positive trend for the scaled union overlap classification average as explained by Table 9 , but is not statistically significant. Since the boosting factor is computed based on the duty cycles of the columns only within the inhibition radius, the full effect of boosting is likely not captured since the baseline inhibition radius is only four.

Table 9. Boosting software sweep

\begin{tabular}{|c|c|c|c|c|}
\hline Maximum boost & SVM Mean \% & SVM STD & SUO Mean \% & SUO STD \\
\hline 1 & 92.91 & 0.33 & 44.56 & 2.74 \\
\hline 1.5 & 92.74 & 0.28 & 44.60 & 2.24 \\
\hline 2 & 92.61 & 0.27 & 43.27 & 2.33 \\
\hline 3 & 92.76 & 0.24 & 46.01 & 2.86 \\
\hline
\end{tabular}




\subsubsection{Minimum Overlap}

Raising the minimum overlap by a fixed value instead of varying based on activation shows an improvement in accuracy, but without the large variation in accuracies between runs as was seen with the variable minimum overlap method. Using a value of three or four provides a nice compromise between accuracy and variation. These results are shown in Table 10.

Table 10. Minimum overlap software sweep

\begin{tabular}{|c|c|c|c|c|}
\hline Minimum overlap & SVM Mean \% & SVM STD & SUO Mean \% & SUO STD \\
\hline 1 & 92.91 & 0.33 & 44.56 & 2.74 \\
\hline 2 & 93.90 & 0.23 & 48.92 & 3.60 \\
\hline 3 & 94.82 & 0.16 & 52.17 & 2.38 \\
\hline 4 & 94.37 & 0.37 & 52.34 & 2.57 \\
\hline 5 & 91.58 & 0.39 & 50.32 & 0.82 \\
\hline 6 & 86.72 & 0.25 & 45.95 & 7.71 \\
\hline 7 & 80.89 & 1.10 & 18.47 & 9.56 \\
\hline 8 & 71.35 & 0.24 & 8.08 & 2.39 \\
\hline 9 & 57.28 & 2.44 & 9.89 & 2.85 \\
\hline 10 & 43.72 & 1.84 & 10.78 & 1.37 \\
\hline
\end{tabular}

\section{$\underline{5.2 .8 \quad \text { Span }}$}

The span setting controls how many different inputs may be to connected to each synapse in a particular column. By restricting the input space to which a column may be connected, each column will be restricted to identifying features in a smaller range. As can be seen in Table 11, this has a positive effect on classification accuracy. However not 
all data will have as much spatial significance as an image like was used in these experiments. Input data without spatial significance would not be likely to benefit from applying a span constraint. The hardware implementation will use a span of 112 since that span shows higher classification and the least variation.

Table 11. Span software sweep

\begin{tabular}{|c|c|c|c|c|}
\hline Span & SVM Mean \% & SVM STD & SUO Mean \% & SUO STD \\
\hline None (entire input) & 92.91 & 0.33 & 44.56 & 2.74 \\
\hline 28 (1 row) & 96.31 & 0.15 & 53.88 & 2.54 \\
\hline $56(2$ rows) & 96.25 & 0.16 & 53.58 & 1.77 \\
\hline $84(3$ rows) & 96.15 & 0.19 & 54.50 & 2.03 \\
\hline $112(4$ rows $)$ & 95.67 & 0.12 & 55.31 & 0.42 \\
\hline 140 (5 rows) & 95.68 & 0.24 & 55.37 & 1.12 \\
\hline $168(6$ rows $)$ & 95.45 & 0.23 & 55.06 & 2.74 \\
\hline
\end{tabular}

\subsubsection{Final Parameters for Hardware Configuration}

Through this analysis, as well as more iterative testing, a final set of parameters were selected as the seed for the hardware testing. These parameters are detailed in Table 12. 
Table 12. Final software experiment parameters

\begin{tabular}{|c|c|}
\hline Parameter & Value \\
\hline Columns & 512 \\
\hline Synapses per column & 48 \\
\hline Permanence threshold & 24 \\
\hline Inhibition radius & 10 \\
\hline Minimum target activated columns & Unused \\
\hline Maximum target activated columns & Unused \\
\hline Maximum boost factor & 3 \\
\hline Minumum overlap & 112 \\
\hline Span & \\
\hline
\end{tabular}

With these final parameters used, the Python model produces the following results show in in Table 13.

Table 13. Final software experiment results

\begin{tabular}{|c|c|c|c|c|c|c|c|c|}
\hline $\begin{array}{c}\text { SVM } \\
\text { Mean } \\
\text { \% }\end{array}$ & $\begin{array}{c}\text { SVM } \\
\text { STD }\end{array}$ & $\begin{array}{c}\text { SUO } \\
\text { Mean } \\
\text { \% }\end{array}$ & $\begin{array}{c}\text { SUO } \\
\text { STD }\end{array}$ & Power & $\begin{array}{c}\text { HTM } \\
\text { time per } \\
\text { sample }\end{array}$ & $\begin{array}{c}\text { Energy } \\
\text { per } \\
\text { sample }\end{array}$ & $\begin{array}{c}\text { SUO time } \\
\text { per } \\
\text { sample }\end{array}$ & $\begin{array}{c}\text { SVM time } \\
\text { per sample }\end{array}$ \\
\hline 95.43 & 0.24 & 66.06 & 2.57 & $12.125 \mathrm{~W}$ & $11.11 \mathrm{~ms}$ & $135 \mathrm{~mJ}$ & $2.06 \mathrm{~ms}$ & $330.6 \mathrm{~ms}$ \\
\hline
\end{tabular}

The classification results show that the original assumption that each of the parameters were independent variables was incorrect since the mean classification accuracy differences are not additive. However, in the case of SUO classification, the final configuration shows the highest classification accuracy of all experiments. The power reported is the difference in power of the system while running the HTM spatial pooler Python model, and the idle power consumption. The HTM time per sample also 
includes the time taken to read and write the input and output vectors to the disk. In the hardware results section, there are more details on how the IO delay is contributing to the processing time. The energy per sample metric is simply the recorded power multiplied by the time per sample. SUO time is the time required to process a single sample by the Perl based SUO script, and SVM time per sample is how long on average the SVM classification algorithm took to classify a single sample.

Table 14 shows the average classification accuracy of the scaled union overlap method versus that of the non-scaled union overlap across all experiments. As seen, there is a measurable difference between the average classification accuracies. The average runtime increase of adding the scaling was $53 \%$.

Table 14. Scaled union overlap versus union overlap

\begin{tabular}{|c|c|c|c|}
\hline SUO Mean \% & SUO STD & UO Mean \% & UO STD \\
\hline 45.00 & 5.21 & 29.47 & 2.25 \\
\hline
\end{tabular}

\subsection{Hardware}

The HDL was written such that each of the previously described parameters are configurable through either instantiation parameters, "localparam" statements, compiler directives, or configuration registers programmed via AXI. Several iterations were performed in both hardware and software to derive a reasonably ideal set of parameters balancing runtime, resource utilization, timing constraints, and classification accuracy. These settings are the ones described in the previous section. The next subsections will provide results of the hardware implementation experiments, and compare the hardware SUO implementation against both the software equivalent and SVM classifiers. 


\subsubsection{Classification Methods Results}

The parameters used to evaluate the hardware implementation are shown in the previous section in Table 14, with two exceptions: The minimum overlap parameter, as well as another parameter referred to as "shift", were swept by the Zynq processor on the FPGA. The shift parameter controls the calculation of the boost factor for each column. As discussed previously, the duty cycle of each column is represented by an 11-bit unsigned integer, and is used to calculate the variable $m_{i}$ in Equation 13, as shown in Equation 16.

$$
m_{i}=\frac{M_{i}}{2^{\text {shift }}}
$$

Where the division operation is implemented as a logical right-shift of the duty cycle by "shift" number of bits. No boosting occurs when shift $=11$, since $m_{i}=0$, and maximum boosting occurs when shift $=0$. Table 15 details the best results for both SUO and SVM classification methods. Again, five separate tests were conducted with each set of parameters, each with a unique RNG seed.

Table 15. SVM vs. hardware SUO (\%)

\begin{tabular}{|c|c|c|c|c|c|}
\hline Method & Mean & STD & Shift & Min overlap & Runtime \\
\hline $\begin{array}{c}\text { SVM } \\
\text { (software) }\end{array}$ & 94.66 & 0.22 & 8 & 1 & $47 \mathrm{~m}, 33 \mathrm{~s}$ \\
\hline $\begin{array}{c}\text { SUO } \\
\text { (hardware) }\end{array}$ & 71.08 & 1.75 & 6 & 5 & $36 \mathrm{~s}$ \\
\hline
\end{tabular}

\subsubsection{Hardware Parameter Sweep}

The shift parameter was swept from 0 through 11 , and minimum activation ranged from 1 through 12 . Tables 16 and 17 highlight the classification accuracies as functions of these parameters. In each column, the highest accuracy is highlighted in 
blue. Accuracy dropped off rapidly when minimum overlap (MO) was greater than 7 , and shift was less than 3, so these values are omitted from the tables.

Table 16. SVM accuracies with hardware vectors (\%)

\begin{tabular}{|c|c|c|c|c|c|c|c|}
\hline Shift & MO 1 & MO 2 & MO 3 & MO 4 & MO 5 & MO 6 & MO 7 \\
\hline 11 (no boosting) & 94.62 & 94.64 & 94.48 & 94.43 & 94.31 & 94.06 & 93.49 \\
\hline 10 & 94.62 & 94.64 & 94.48 & 94.43 & 94.31 & 94.06 & 93.49 \\
\hline 9 & 94.64 & 94.65 & 94.46 & 94.45 & 94.31 & 94.00 & 93.49 \\
\hline 8 & 94.66 & 94.65 & 94.44 & 94.45 & 94.27 & 93.97 & 93.45 \\
\hline 7 & 94.55 & 94.53 & 94.39 & 94.41 & 94.21 & 94.10 & 93.47 \\
\hline 6 & 94.55 & 94.62 & 94.45 & 94.36 & 94.28 & 93.98 & 93.62 \\
\hline 5 & 94.55 & 94.42 & 94.34 & 94.33 & 94.16 & 93.83 & 93.55 \\
\hline 4 & 93.64 & 93.72 & 93.84 & 93.70 & 93.39 & 93.22 & 92.78 \\
\hline 3 (most aggressive boosting) & 93.36 & 93.26 & 92.87 & 92.86 & 92.83 & 93.01 & 92.84 \\
\hline
\end{tabular}

Table 17. SUO accuracies with hardware vectors (\%)

\begin{tabular}{|c|c|c|c|c|c|c|c|}
\hline Shift & MO 1 & MO 2 & MO 3 & MO 4 & MO 5 & MO 6 & MO 7 \\
\hline 11 (no boosting) & 70.57 & 69.97 & 69.99 & 70.29 & 69.50 & 69.45 & 68.74 \\
\hline 10 & 70.57 & 69.97 & 69.99 & 70.29 & 69.50 & 69.45 & 68.74 \\
\hline 9 & 70.55 & 69.96 & 69.95 & 70.20 & 69.50 & 69.49 & 68.72 \\
\hline 8 & 70.47 & 69.98 & 69.87 & 70.19 & 69.55 & 69.36 & 68.74 \\
\hline 7 & 70.40 & 69.89 & 69.84 & 70.09 & 69.41 & 69.74 & 68.80 \\
\hline 6 & 70.28 & 69.90 & 70.06 & 69.88 & 70.08 & 70.28 & 69.24 \\
\hline 5 & 70.73 & 70.23 & 70.67 & 70.62 & 70.22 & 71.08 & 70.36 \\
\hline 4 & 70.38 & 69.90 & 70.32 & 69.94 & 68.97 & 68.22 & 65.73 \\
\hline 3 (most aggressive boosting) & 63.23 & 62.53 & 62.81 & 62.26 & 61.69 & 62.95 & 60.05 \\
\hline
\end{tabular}


The data in Table 16 suggests there is not much of a benefit to boosting in the spatial pooler with respect to using SVM classification. However, Table 17 shows there does seem to be a slight positive correlation between boosting and accuracy when using SUO. In fact, for this test set, across all values of minimum overlap, a shift of five produces the best results. Figure 26 provides a surface plot of the data from Table 17 .

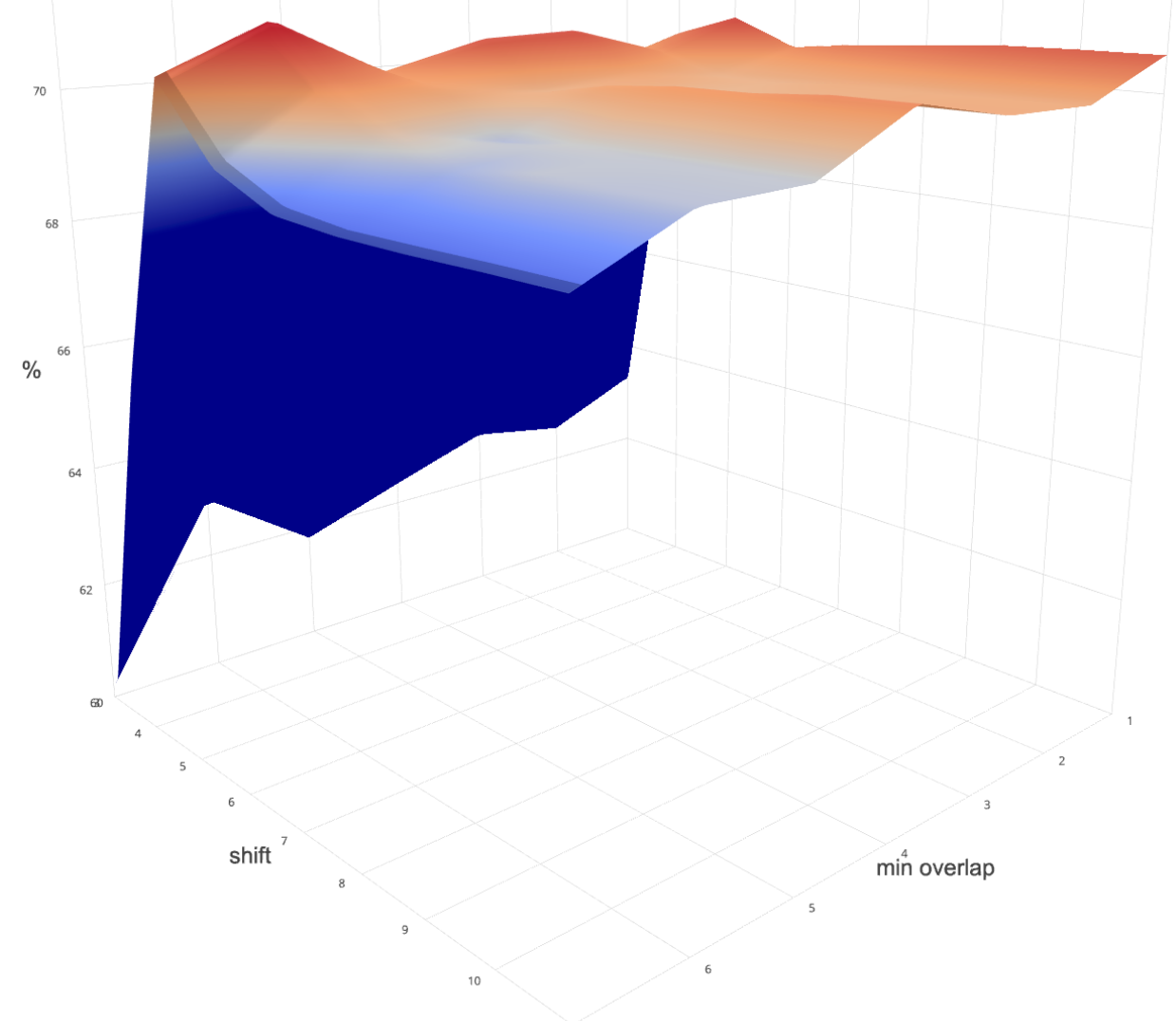

Figure 26. SUO accuracy surface plot

\subsubsection{FPGA Resources}

Three experiments were performed in order to determine how many hardware resources are being utilized for these three separate functions:

1. The core HTM SP algorithm 


\section{Boosting}

\section{Scaled Union Overlap}

Three versions of the HDL design were synthesized, placed, and then routed. After placing the design, reports are written by the Vivado suite detailing how many of each type of hardware blocks were used to implement the logic. The htm_core Verilog module was written such that the boosting and classification functions can be included or excluded independently based on the definition of pre-processor macros. For the first experiment, both the boosting and classification functions were enabled to get a maximum utilization. The other two experiments enabled only one of the two functions, boosting or classification, and the results were recorded. The decrease in resources required when each function was removed represents can be seen below in Figure 27. The full utilization reports can be found in Appendix B. 

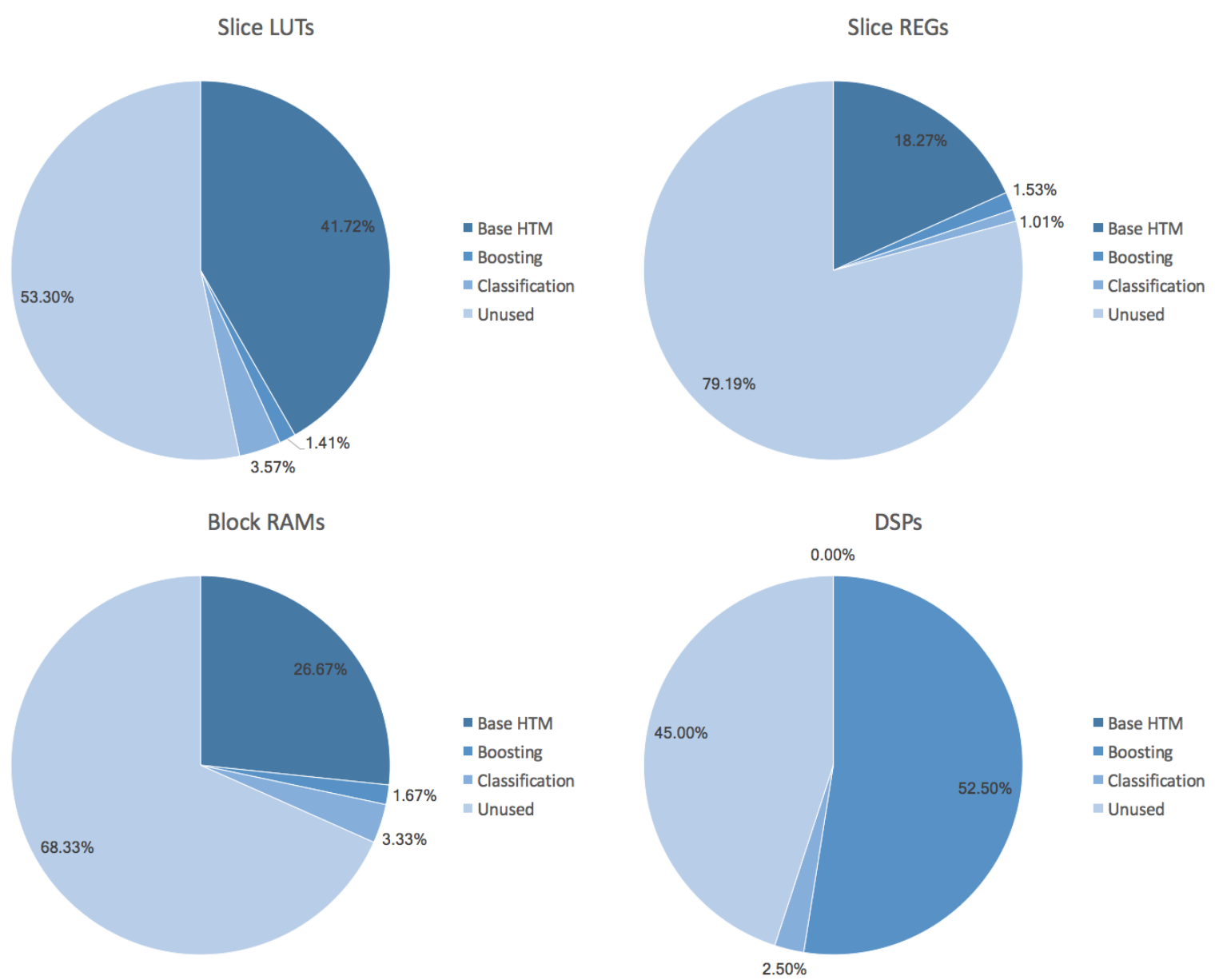

Figure 27. Slice LUTs utilization

The number of slice Look-Up Tables (LUTs) reflects the combinatorial logic operations required to implement each function. The slice registers are the number of single bit memory elements used to preserve state. The block RAMs refers to the number of discreet 36 KB SRAM macros used. The Digital Signal Processors (DSPs) are logic blocks used to perform common complex arithmetic functions such as single cycle addition or multiplication.

This data shows that the block RAMs and slice logic are dominantly used to implement the core spatial pooling algorithm. However, the DSP usage is almost entirely attributed to the boosting functionality. This is because DSPs are being used to do the 
arithmetic operations shown in Figure 23 to generate the boosting factors from Equation 15. Classification also requires some DSPs, but the squaring functionality, which is the most logically complex operation classification uses, was offloaded to a block RAM.

From a utilization perspective, it should be reasonable to expect to be able to implement two htm_cores on this programmable SoC.

\subsection{Comparison}

This section summarizes and compares metrics between the software and hardware spatial poolers, as well as the hardware SUO implementation against the SVM and SUO classifiers.

\subsubsection{Spatial Poolers}

As expected, the hardware implementation improved speed and power efficiency. Table 18 summarizes the major metrics used to characterize the spatial pooler.

Table 18. Hardware versus software SP

\begin{tabular}{|l|c|c|c|}
\hline SP Type & $\begin{array}{c}\text { Accuracy } \\
\text { (SVM) }\end{array}$ & $\begin{array}{c}\text { Speed } \\
\text { (us per vector) }\end{array}$ & $\begin{array}{c}\text { Efficiency } \\
\text { (mJ per vector) }\end{array}$ \\
\hline Software & 95.43 & 11114.43 & $135 \mathrm{~mJ}$ \\
\hline Hardware & 94.66 & 85.71 & $154 \mathrm{uJ}$ \\
\hline Difference & $-0.81 \%$ & $130 \mathrm{X}$ speedup & $877 \mathrm{X}$ reduction \\
\hline
\end{tabular}

The hardware implementation shows significant improvements to both speed and power efficiency, with a slight degradation in accuracy. A small difference in accuracy is expected between implementations since each uses a different method for generating pseudo-random numbers during initialization. 


\subsubsection{Classifiers}

This section documents the results of comparing four classifiers:

1. The support vector machine classifier, which provides a high classification accuracy at the cost of speed and power.

2. The scaled overlap method, which is the simplest yet least accurate method, and

3. Hardware implementations of scaled union overlap. Both the third and fourth classifiers provide an intermediate level of accuracy at the expense of a slight increase in complexity.

Table 19 provides the metrics of interest for these four methods. The vectors used to characterize the classifiers are those produced by the hardware SP with the shift parameter set to 5. Each of these values represents the average across all of these test cases.

Table 19. Classifier metric comparison

\begin{tabular}{|c|c|c|c|}
\hline Classifier & Accuracy (\%) & $\begin{array}{c}\text { Speed } \\
\text { (us per vector) }\end{array}$ & $\begin{array}{c}\text { Energy consumption } \\
\text { (mJ per vector) }\end{array}$ \\
\hline SVM (software) & 94.17 & $300.1 \mathrm{~ms}$ & 3637.50 \\
\hline $\begin{array}{c}\text { Union overlap } \\
\text { (software) }\end{array}$ & 63.93 & $1.54 \mathrm{~ms}$ & 18.67 \\
\hline $\begin{array}{c}\text { Scaled union } \\
\text { overlap (software) }\end{array}$ & 70.62 & $2.34 \mathrm{~ms}$ & 28.37 \\
\hline $\begin{array}{c}\text { Scaled union } \\
\text { overlap } \\
\text { (hardware) }\end{array}$ & 70.65 & $0.1 \mathrm{~ms}$ & 0.18 \\
\hline
\end{tabular}

This table highlights the main benefits of implementing a classifier on the hardware, as opposed to an offline software equivalent. However, it is also possible that the SUO could be performed on-chip by the Zynq processor instead of offline on a 
separate processor. This would have the benefit of providing real-time online classification, without the additional complexity to the programmable logic. However, there would likely be both a speed as well as efficiency penalty in doing this.

The Intel Core i7 required roughly 6.8 million clock cycles to perform one classification. Assuming the same number of cycles would be required to do the same operation on the Zynq, at $100 \mathrm{MHz}$, this same operation would take approximately 67.9 ms. Also, at the nominal 1.796 Watts that the FPGA consumes, this would translate to an estimated $121.9 \mathrm{~mJ}$ per classification. Thus, moving the classification algorithm into the programmable logic could theoretically speed performance, and consequently increase energy efficiency as well, both by $680 \mathrm{X}$. 


\section{CHAPTER 6: CONCLUSION}

\subsection{Summary}

This thesis introduced a novel implementation of the HTM spatial pooler on a programmable SoC. The design was based mostly on the work described by Zyarah and Kudithipudi titled "Reconfigurable Hardware Architectures of the Spatial Pooler for Hierarchical Temporal Memory" [20], with two main contributions. The first being the introduction of a method for boosting unused columns, and the second adding a modified version of the classification algorithm described by Ahmad and Hawkins in their work "How do neurons operate on sparse distributed representations? A mathematical theory of sparsity, neurons and active dendrites" [21], both to the programmable logic. The efficacy and benefits were explored, and a mild benefit was found from the addition of boosting, and a sizable benefit in both speed and power efficiency from the classification being moved to the programmable logic.

\subsection{Future Work}

This thesis explored the effectiveness of the boosting functionality by classifying image data from the MNIST database. A modest benefit was shown to enabling this functionality for this test case. Further work may be done to evaluate the benefits of this method of boosting by testing datasets with different properties. The MNIST dataset only requires classification into ten different bins. It's possible that increasing the number of bins might highlight the benefits of higher SDR entropy which boosting provides. 
A large portion of the hardware resources were dedicated to storing and processing the addresses which map the synapses back to the input space. If restricting the range of inputs is not required, another solution to producing these addresses is to dynamically create them during each pooling iteration with a linear feedback shift register, or some other pseudo-random number generator. With an LFSR, the state of the shift register could be reset to the same value at the beginning of the pooling algorithm, thus ensuring the same pattern of addresses would be repeated each iteration. In this case, a saving of 36 kilo-bytes of SRAM resources can be achieved.

The spatial pooling algorithm performs only one part of the HTM model. The idea of the synthetic synapse and processing techniques presented here could also be extended to include temporal pooling. However, since there are several cells per column, the processing bandwidth and storage requirements would need to be higher than that of spatial pooling. In this case, the previous suggestion above to recreate the indexed addresses during each iteration could potentially provide more of an area benefit, at the cost of higher energy consumption per sample. 


\section{REFERENCES}

[1] Fast, E. and Horvitz, E., 2017, February. Long-Term Trends in the Public Perception of Artificial Intelligence. In $A A A I$ (pp. 963-969).

[2] Ovtcharov, Kalin, et al. "Accelerating deep convolutional neural networks using specialized hardware." Microsoft Research Whitepaper 2.11 (2015).

[3] Demirci, Mehmet. "A survey of machine learning applications for energy-efficient resource management in cloud computing environments." Machine Learning and Applications (ICMLA), 2015 IEEE 14th International Conference on. IEEE, 2015.

[4] Abadi, Martín, et al. "Tensorflow: a system for large-scale machine learning." OSDI. Vol. 16. 2016.

[5] Shi, Shaohuai, et al. "Benchmarking state-of-the-art deep learning software tools." Cloud Computing and Big Data (CCBD), 2016 7th International Conference on. IEEE, 2016.

[6] Schneider, David. "Deeper and cheaper machine learning [top tech 2017]." IEEE Spectrum 54.1 (2017): 42-43.

[7] Chen, Yunji, et al. "Dadiannao: A machine-learning supercomputer." Proceedings of the 47th Annual IEEE/ACM International Symposium on Microarchitecture. IEEE Computer Society, 2014.

[8] Hawkins, Jeff, Subutai Ahmad, and Donna Dubinsky. "Hierarchical temporal memory including HTM cortical learning algorithms." Technical report, Numenta, Inc, Palo Alto http://www.numenta.com/htmoverview/education/HTM CorticalLearningAlgorith ms.pdf (2011).

[9] IEEE. (2001). IEEE Standard Verilog Hardware Description Language (IEEE Std 1364-2001). New York, NY: IEEE Computer Society 
[10] Digilent. ZYBO FPGA Board Reference Manual, April, 2016. https://reference.digilentinc.com/_media/zybo:zybo_rm.pdf

[11] LeCun, Yann. "The MNIST database of handwritten digits." http://yann.lecun.com/exdb/mnist/(1998).

[12] C.-C. Chang and C.-J. Lin. LIBSVM: a library for support vector machines. ACM Transactions on Intelligent Systems and Technology, 2:27:1--27:27, 2011.

[13] Williams, Stephen. "Icarus verilog." On-line: http://iverilog. icarus. com (2006).

[14] Bybell, Tony. "GtkWave electronic waveform viewer." (2010).

[15] P3 International. P4400 Kill A Watt Operation Manual, February, 2014. A.

[16] Hawkins, Jeff, and Sandra Blakeslee. On intelligence: How a new understanding of the brain will lead to the creation of truly intelligent machines. Macmillan, 2007.

[17] Barth, Alison L., and James FA Poulet. "Experimental evidence for sparse firing in the neocortex." Trends in neurosciences 35.6 (2012): 345-355.

[18] Eshghi, Kave, and Mehran Kafai. "Support vector machines with sparse binary highdimensional feature vectors." Hewlett Packard Labs, Palo Alto, CA, USA, Tech. Rep. HPE-2016-30 (2016): 1-10.

[19] Streat, Lennard, Dhireesha Kudithipudi, and Kevin Gomez. "Non-volatile hierarchical temporal memory: Hardware for spatial pooling." arXiv preprint arXiv:1611.02792 (2016).

[20] Zyarah, Abdullah M., and Dhireesha Kudithipudi. "Reconfigurable hardware architecture of the spatial pooler for hierarchical temporal memory." System-onChip Conference (SOCC), 2015 28th IEEE International. IEEE, 2015.

[21] Ahmad, Subutai, and Jeff Hawkins. "How do neurons operate on sparse distributed representations? A mathematical theory of sparsity, neurons and active dendrites." arXiv preprint arXiv:1601.00720 (2016).

[22] Mnatzaganian, James, Ernest Fokoué, and Dhireesha Kudithipudi. "A mathematical formalization of hierarchical temporal memory's spatial pooler." Frontiers in Robotics and AI 3 (2017): 81. 
[23] Cui, Yuwei, Subutai Ahmad, and Jeff Hawkins. "The HTM spatial pooler —a neocortical algorithm for online sparse distributed coding." Frontiers in computational neuroscience 11 (2017): 111.

[24] Xilinx. Zynq-7000 SoC Data Sheet: Overview, July, 2018. https://www.xilinx.com/support/documentation/data_sheets/ds190-Zynq-7000Overview.pdf

[25] George, Maria, and Peter Alfke. "Linear feedback shift registers in Virtex devices." Xilinx application note XAPP210 (2007). 
APPENDIX A 


\section{Macbook Pro Specifications}

A late 2016 Macbook Pro with Touchbar was used for running all simulations, as well as creating and testing of support vector machines. This machine had the following specifications:

Processor

Intel 2.9 GHz Core i7 (I7-6920HQ), 4-cores, 256 KB L2, 8MB L3

DRAM

2 DIMMs, 8 GB (16 GB total) LPDDR3 @ $2133 \mathrm{MHz}$

Storage

512 GB SSD, PCI-Express 3.0

Operating System

macOS 10.13.6 
APPENDIX B 


\section{Utilization Reports}

Table B.1 All functions enabled LUT utilization

\begin{tabular}{|c|c|c|c|c|}
\hline Site Type & Used & Fixed & Available & Util\% \\
\hline Slice LUTs & 8219 & 0 & 17600 & 46.7 \\
\hline LUT as Logic & 7374 & 0 & 17600 & 41.9 \\
\hline LUT as Memory & 845 & 0 & 6000 & 14.08 \\
\hline LUT as Distributed RAM & 424 & 0 & & \\
\hline LUT as Shift Register & 421 & 0 & & \\
\hline Slice Registers & 7324 & 0 & 35200 & 20.81 \\
\hline Register as Flip Flop & 7324 & 0 & 35200 & 20.81 \\
\hline Register as Latch & 0 & 0 & 35200 & 0 \\
\hline F7 Muxes & 223 & 0 & 8800 & 2.53 \\
\hline F8 Muxes & 74 & 0 & 4400 & 1.68 \\
\hline
\end{tabular}

Table B.2 All functions enabled RAM utilization

\begin{tabular}{|c|c|c|c|c|}
\hline Site Type & Used & Fixed & Available & Util\% \\
\hline Block RAM Tile & 18 & 0 & 60 & 30 \\
\hline RAMB36/FIFO* & 17 & 0 & 60 & 28.33 \\
\hline FIFO36E1 only & 3 & & & \\
\hline RAMB36E1 only & 14 & & & \\
\hline RAMB18 & 2 & 0 & 120 & 1.67 \\
\hline RAMB18E1 only & 2 & & & \\
\hline
\end{tabular}


Table B.3 All functions enabled DSP utilization

\begin{tabular}{|c|c|c|c|c|}
\hline Site Type & Used & Fixed & Available & Util\% \\
\hline DSPs & 44 & 0 & 80 & 55 \\
\hline DSP48E1 only & 44 & & & \\
\hline
\end{tabular}

Table B.4 Boosting disabled LUT utilization

\begin{tabular}{|c|c|c|c|c|}
\hline Site Type & Used & Fixed & Available & Util\% \\
\hline Slice LUTs & 7970 & 0 & 17600 & 45.28 \\
\hline LUT as Logic & 7125 & 0 & 17600 & 40.48 \\
\hline LUT as Memory & 845 & 0 & 6000 & 14.08 \\
\hline LUT as Distributed RAM & 424 & 0 & & \\
\hline LUT as Shift Register & 421 & 0 & & \\
\hline Slice Registers & 6784 & 0 & 35200 & 19.27 \\
\hline Register as Flip Flop & 6784 & 0 & 35200 & 19.27 \\
\hline Register as Latch & 0 & 0 & 35200 & 0 \\
\hline F7 Muxes & 206 & 0 & 8800 & 2.34 \\
\hline F8 Muxes & 71 & 0 & 4400 & 1.61 \\
\hline
\end{tabular}


Table B.5 Boosting disabled RAM utilization

\begin{tabular}{|c|c|c|c|c|}
\hline Site Type & Used & Fixed & Available & Util\% \\
\hline Block RAM Tile & 17 & 0 & 60 & 28.33 \\
\hline RAMB36/FIFO* & 16 & 0 & 60 & 26.67 \\
\hline FIFO36E1 only & 2 & & & \\
\hline RAMB36E1 only & 14 & & & \\
\hline RAMB18 & 2 & 0 & 120 & 1.67 \\
\hline FIFO18E1 only & 1 & & & \\
\hline RAMB18E1 only & 1 & & & \\
\hline
\end{tabular}

Table B.6 Boosting disabled DSP utilization

\begin{tabular}{|c|c|c|c|c|}
\hline Site Type & Used & Fixed & Available & Util\% \\
\hline DSPs & 2 & 0 & 80 & 2.5 \\
\hline DSP48E1 only & 2 & & & \\
\hline
\end{tabular}

Table B.7 Classification disabled LUT utilization

\begin{tabular}{|c|c|c|c|c|}
\hline Site Type & Used & Fixed & Available & Util\% \\
\hline Slice LUTs & 7591 & 0 & 17600 & 43.13 \\
\hline LUT as Logic & 6746 & 0 & 17600 & 38.33 \\
\hline LUT as Memory & 845 & 0 & 6000 & 14.08 \\
\hline LUT as Distributed RAM & 424 & 0 & & \\
\hline LUT as Shift Register & 421 & 0 & & \\
\hline Slice Registers & 6970 & 0 & 35200 & 19.8 \\
\hline Register as Flip Flop & 6970 & 0 & 35200 & 19.8 \\
\hline Register as Latch & 0 & 0 & 35200 & 0 \\
\hline F7 Muxes & 148 & 0 & 8800 & 1.68 \\
\hline F8 Muxes & 61 & 0 & 4400 & 1.39 \\
\hline
\end{tabular}


Table B.8 Classification disabled RAM utilization

\begin{tabular}{|c|c|c|c|c|}
\hline Site Type & Used & Fixed & Available & Util\% \\
\hline Block RAM Tile & 16.5 & 0 & 60 & 27.5 \\
\hline RAMB36/FIFO* & 16 & 0 & 60 & 26.67 \\
\hline FIFO36E1 only & 3 & & & \\
\hline RAMB36E1 only & 13 & & & \\
\hline RAMB18 & 1 & 0 & 120 & 0.83 \\
\hline RAMB18E1 only & 1 & & & \\
\hline
\end{tabular}

Table B.9 Classification disabled DSP utilization

\begin{tabular}{|c|c|c|c|c|}
\hline Site Type & Used & Fixed & Available & Util\% \\
\hline DSPs & 42 & 0 & 80 & 52.5 \\
\hline DSP48E1 only & 42 & & & \\
\hline
\end{tabular}

